\title{
A Revised Sensitivity Model for Cassini INMS: Results at Titan
}

\author{
B.D. Teolis ${ }^{1}$ • H.B. Niemann ${ }^{2}$ - J.H. Waite ${ }^{1}$ - D.A. Gell ${ }^{1}$. R.S. Perryman ${ }^{1}$. \\ W.T. Kasprzak ${ }^{2}$ - K.E. Mandt ${ }^{1}$ - R.V. Yelle ${ }^{3}$ A.Y. Lee ${ }^{4}$ - F.J. Pelletier ${ }^{4}$ G.P. Miller ${ }^{1}$. \\ D.T. Young ${ }^{1}$ J.M. Bell ${ }^{1,5}$ • B.A. Magee ${ }^{1}$ - E.L. Patrick ${ }^{1}$ - J. Grimes ${ }^{1}$ - G.G. Fletcher ${ }^{1}$. \\ V. Vuitton ${ }^{6}$
}

Received: 1 August 2014 / Accepted: 30 December 2014 / Published online: 20 January 2015

(C) The Author(s) 2015. This article is published with open access at Springerlink.com

\begin{abstract}
Cassini Ion Neutral Mass Spectrometer (INMS) measurements from roughly a hundred Titan encounters over the Cassini mission yield neutral and ion densities systematically lower, by factors approximately 2 to 3, than estimates from several other spacecraft systems, including the Attitude and Articulation Control System, and Navigation system. In this paper we present a new INMS instrument sensitivity model, obtained by re-analyzing (1) the capture and transmission of neutral gas through the instrument, and (2) the detector gain reduction during pre-launch testing. By correcting for an under-estimation of gas leakage out of the instrument into space by the original calibration model, and adjusting for the gain change, the new model brings INMS densities into much closer agreement with the other Cassini systems. Accordingly, the INMS ion densities are revised upward by a constant detector sensitivity correction factor of $1.55 \pm 21 \%$, while the neutral sensitivities have a complex instrument pointing direction dependence, due (mostly) to the effect of the INMS vent and antechamber-to-closed source tube. In the special case of on-ram pointing the neutral densities are revised upward by a constant factor of $2.2 \pm 23 \%$. The corrected neutral and ion sensitivities given here are applicable to all previously published INMS results at Titan, Enceladus and elsewhere in the Saturn system. The new model gives reliable densities at high ram angles, in some cases above 90 degrees, thereby expanding the list of Titan flybys from which INMS densities may be extracted. We apply the model to obtain
\end{abstract}

B.D. Teolis

ben.teolis@swri.org

1 Space Science Division, Southwest Research Institute, 6220 Culebra Road, San Antonio, TX 78238, USA

2 NASA Goddard Space Flight Center, Greenbelt, MD 20771, USA

3 Department of Planetary Sciences, University of Arizona, Tucson, AZ 85721, USA

4 Jet Propulsion Laboratory, California Institute of Technology, Mail Stop 230-104, Pasadena, CA 91109, USA

5 National Institute of Aerospace, 100 Exploration Way, Hampton, VA 23666, USA

6 Université de Grenoble Alpes/CNRS, Institut de Planétologie et d'Astrophysique de Grenoble, Grenoble 38000, France 
accurate densities from several off-ram Titan flybys which gave unusual neutral density vs. altitude profiles, or unreasonably high densities, with the original calibration.

Keywords Cassini · Ion Neutral Mass Spectrometer · Cassini INMS · Instrument sensitivity · Titan · Saturn · Enceladus · Monte Carlo simulations · Gas conductance · Atmospheres · Attitude and articulation control · Thrusters · Spacecraft navigation · Instrument calibration

\section{Introduction}

The primary scientific objectives of the Cassini Ion Neutral Mass Spectrometer (INMS) investigation are: (1) to characterize the composition, structure, and chemical behavior of Titan's upper atmosphere and its interaction with Saturn's magnetosphere; and (2) to investigate the neutral and plasma environments of the rings and icy satellites and their magnetospheric interactions (Waite et al. 2004). Careful determination of the gas densities of the environment is necessary for the proper execution of these objectives. The ion density extraction process has been discussed in detail by Mandt et al. (2012) for ram pointed flybys, and many other aspects of the INMS calibration and instrument response to ions and neutrals have now also been addressed in literature [see, e.g., Cui et al. 2009; Gell and Waite 2015; Magee et al. 2009; Perry et al. 2010; Teolis et al. 2010; Vuitton et al. 2008; Waite et al. 2004], including (1) compositional extraction from mass spectra, (2) wall adsorption of 'sticky' gases such as $\mathrm{H}_{2} \mathrm{O}$ and $\mathrm{NH}_{3}$, (3) thruster firing contamination, (4) radiation background, (5) high-pressure background effects, (6) residual gas 'tails', (7) highsensitivity detector saturation, and (8) on-going work to refine the instrument calibration with the ground-based refurbished engineering unit. However the systematic difference between the INMS neutral and ion densities with other Cassini systems-which has been become apparent during the last decade over more than 100 Titan flybys-has not yet been addressed.

The INMS measurements of Titan's atmospheric neutral mass density were found to be systematically lower than that measured by the Cassini Attitude and Articulation Control System (AACS) and by Navigation (NAV), both of which use spacecraft drag data and aerodynamic models of the spacecraft to derive mass density [as reported by the Titan Atmospheric Working Group [TAMWG], Lee and Hanover 2005]. RPWS Langmuir probe electron densities can serve as a proxy for total ion densities, while ion densities can be retrieved from CAPS-IBS data (Crary et al. 2009). Comparisons of these sources of ion densities also show systematic differences with the ion densities measured by INMS. We have traced the differences between INMS neutral density measurements and other spacecraft systems in part to gas escape from the "closed" source (CS) through leakage pathways not accounted for in the original INMS calibration model. The escape competes with the ram dynamic pressure entering the INMS, yielding lower CS gas densities than indicated by the original calibration, and resulting in a lower INMS neutral gas sensitivity estimate. Therefore the INMS original calibration model that assumed a sealed CS—used until now to relate the CS density to the ambient density — must be replaced by a more accurate approach. This work describes in-flight density comparisons that lead to a new formulation of the ram enhancement factor in the INMS ion sources and, accordingly, a re-estimation of the instrument sensitivity versus spacecraft speed and pointing. We have also re-analyzed the INMS detector gain, and find that the gain reduction which occurred during pre-launch 
characterization testing (Waite et al. 2004), had not been accounted for in the original calibration model. This gain reduction contributes to the reduced INMS sensitivity estimate in the model presented here. The new calibration model brings Cassini INMS results into agreement with the other Cassini systems on the Titan ion and neutral atmospheric densities, and enables extraction of Titan neutral density profiles from several previous flybys that yielded unusual and unreasonable densities using the original calibration model.

The paper is organized as follows. In the main text we limit the discussion to the key issues, i.e., a basic explanation of the new calibration model, its origin and application, and key equations (Sect. 3), the discussion of the detector sensitivity correction (Sect. 4), results at Titan (Sect. 5), comparison of INMS to other Cassini systems (Sect. 6), and possible Titan science implications (Sect. 7). Appendix A gives the full derivation of the new calibration model, while the remaining Appendices address several other relevant questions: the inflight model calibration (Appendix B), empirical fitting functions useful for applying the new model (Appendix C), spacecraft shadowing effects (Appendix D), comparison against the original INMS calibration model (Appendix E), Monte Carlo simulations of gas flow through the INMS (Appendix F), and thruster firing and inter-molecular collisional effects (Appendix G).

\section{Instrument Description}

The INMS (Fig. 1) is a mass spectrometer described in detail by Waite et al. (2004). The instrument is a quadrupole mass spectrometer (Fig. 2) with two source inlets: (1) a closed source (CS) and antechamber for measurement of neutral gas; and (2) an open source (OS) for the measurement of ions and reactive neutrals without wall collisions with the instrument

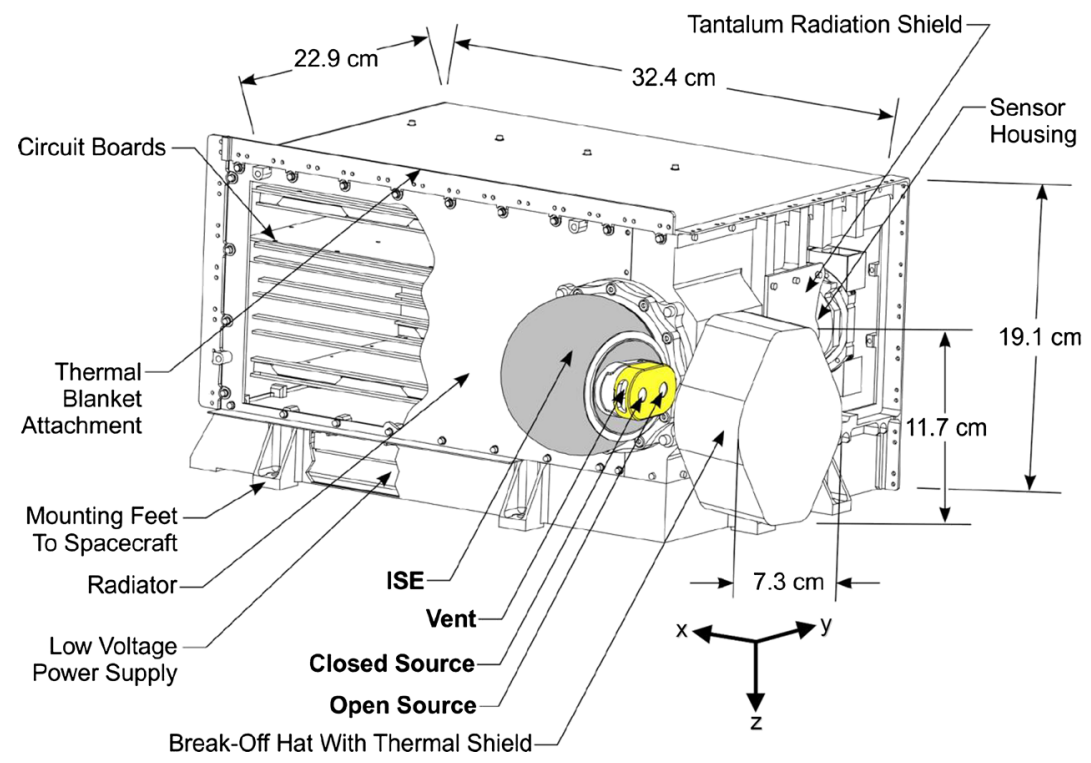

Fig. 1 Drawing of the INMS, showing (in color) the ISE and CS and OS inlet apertures, and vent, in relation to (no color) the instrument and electronics housing. The Break-Off Hat was jettisoned after the launch of Cassini 
Fig. 2 Schematic showing the main components of the INMS. The ram factor model considers the sections above the quadrupole mass analyzer within the ion source enclosure (ISE not shown)

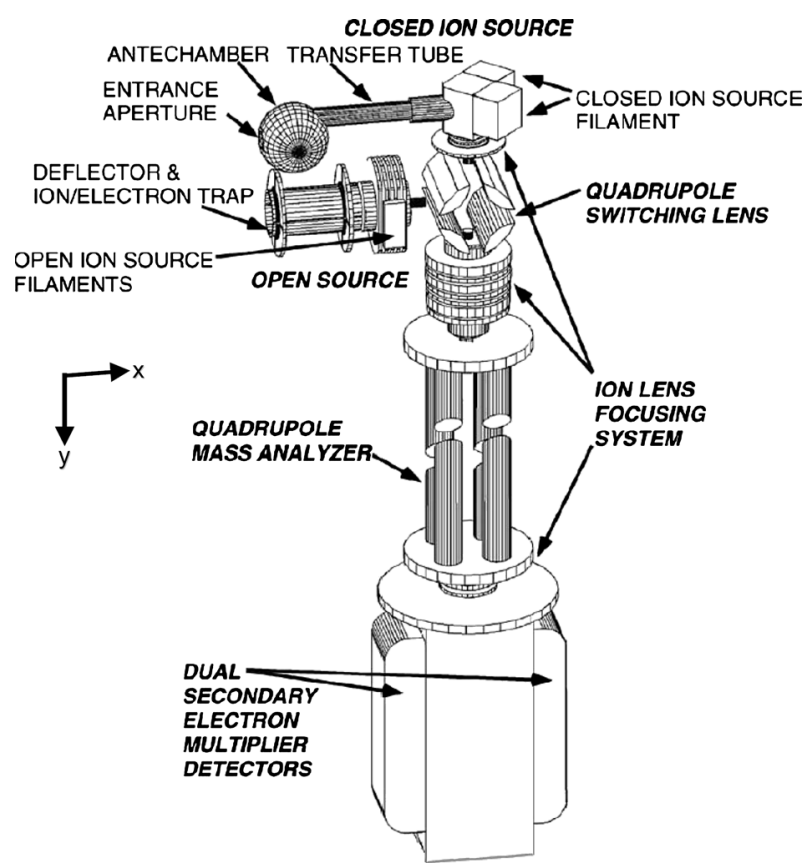

interior (Fig. 3). The sources are housed in an Ion Source Enclosure (ISE). In flight, measurements alternate in a programmable fashion between the two sources. Gas enters the CS inlet through the entrance aperture as a well collimated molecular beam since the relative gas-spacecraft speed is typically much greater than the molecule thermal speeds. Molecule velocities are isotropized and slowed (thermally accommodated) by colliding (roughly 120 times) with the walls of the inlet's antechamber and antechamber-to-CS tube (Teolis et al. 2010) before passing through the tube to the CS ionization region (Fig. 3, labeled with density $n_{s}$ ). The resulting density enhancement in the antechamber and CS is a function of the ram angle between the spacecraft velocity vector and the entrance aperture normal. A typical Titan flyby at $6 \mathrm{~km} / \mathrm{s}$ produces a ram factor of $\sim 71$ relative to the density for a spacecraft at rest if one neglects the leakage paths out of the CS. Scaling the ram factor by $\sqrt{T_{a} / T_{s}}$ yields the CS to ambient density ratio at the CS and ambient gas temperatures $T_{s}$ and $T_{a}$ (see Eq. (A.17)). The side vent (Figs. 1 and 3) limits the accumulation of residual gas inside the instrument, and constitutes a major leakage pathway out of the instrument (Table 1). Neutral gas is ionized in the CS using an electron beam (commandable to $\sim 25$ or $70 \mathrm{eV}$ ) generated by one of two redundant hot filaments. The OS inlet is located below the CS inlet, and consists of an entrance aperture and ion beam collimator. In-flight OS measurement of ambient ion densities is described by Mandt et al. (2012). Positive ions from the CS or OS are extracted into the electrostatic quadrupole lens (Fig. 3), which is used as a deflector to switch ion trajectories between the two sources (Mahhafy and Lai 1990). The quadrupole lens voltages are set to transfer the ions into the analyzer ion focus lenses and in turn into the quadrupole mass analyzer (QMA). The voltage setting on the ion focus lenses can increase or reduce the sensitivity by affecting how well the beam of ionized neutrals is focused into the mass analyzer.

The QMA selects the mass-to-charge ratio $(\mathrm{m} / \mathrm{q})$ in the range of 0.5 to 8.5 and 11.5 to $99.5 \mathrm{u}$ per charge, by way of a quadrupole radio frequency mass filter. The detector fore 


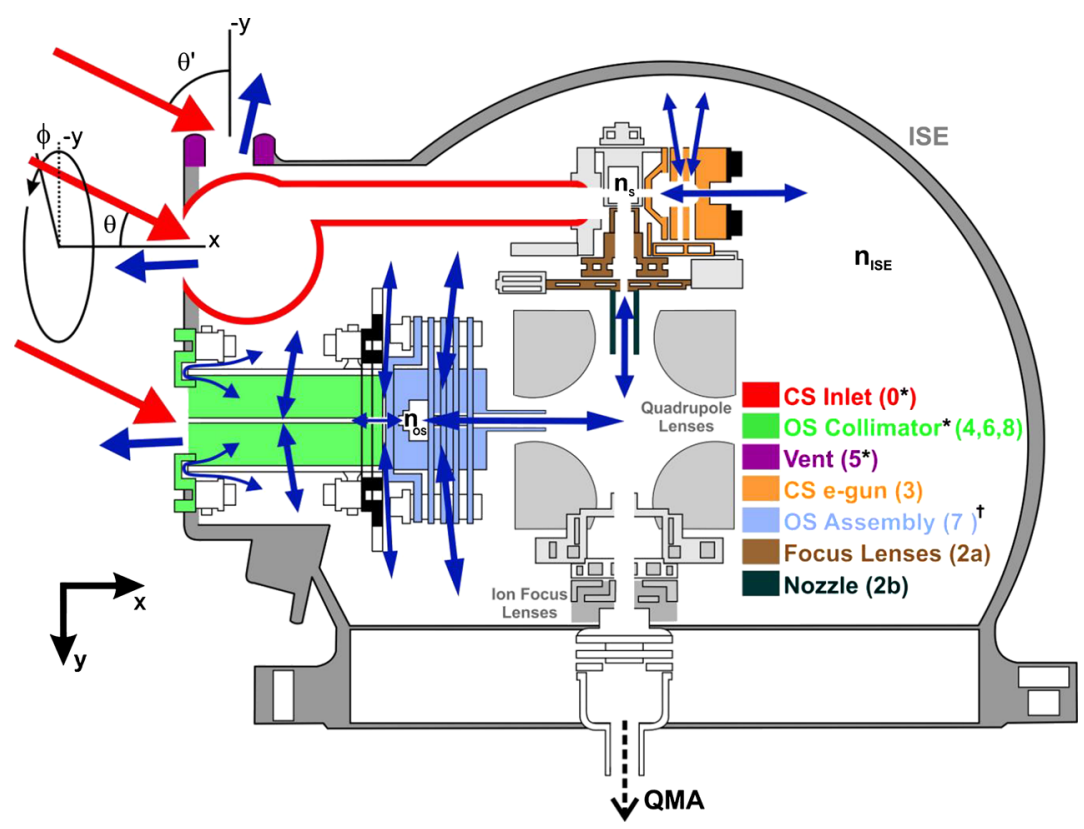

Fig. 3 Cross section diagram (to scale) of the INMS ion source enclosure (ISE, shown in color in Fig. 1) with ion sources and gas inlet system shown, together with the azimuthal $(\phi), \operatorname{ram}(\theta)$ and vent $\operatorname{ram}\left(\theta^{\prime}\right)$ angles, spacecraft coordinates axes, gas flows (red arrows: ram flux, blue arrows: thermalized flows), and the approximate locations of densities $n_{\mathrm{S}}, n_{\mathrm{OS}}$, and $n_{\mathrm{ISE}}$. Conduction paths considered in the ram factor model are colored as indicated by the legend, with number-letter notation (Appendix A) in parentheses. Asterisks: effective area estimated by Monte Carlo simulations. Dagger: effective area estimated by fit to Cassini T85 and T88 data. For remaining pathways, we estimated the effective areas analytically. For clarity, leakage through both CS electron guns is shown here as a single conduction pathway: see Fig. 4 for multiple pathways considered in the CS e-gun conductance calculation. Grey colored components are neglected in the model, since these sections are treated as having either (i) negligible (e.g., CS enclosure around $n_{s}$, ion focus lenses \& QMA), or (ii) maximum (quadrupole lenses) conductance

optics consists of three lenses: the Einzel, Mask I and Mask II, which together focus the ions into the detectors. The lens voltage settings can modify the INMS sensitivity depending on how well they focus the beam exiting the QMA into the detectors. Two secondary electron multipliers detect the resulting ion beam operating in a pulse counting mode. These detectors differ in effective sensitivity, with the lower sensitivity detector operating off of secondary electrons produced from the adjacent higher sensitivity detector (Waite et al. 2004). INMS neutral species-dependent sensitivity factors $s_{f}$ (for species $f$ ) are calibrated on the ground by seven factors: (1) the electron impact efficiency in the sources as determined by the ionization cross sections of each neutral species; (2) the efficiency for extraction of ions from the CS or OS into the quadrupole lens; (3) the transmission bandpass of the quadrupole switching lens; (4) the degree of focusing in the ion focus lenses into the mass analyzer; (5) the QMA transmission; (6) the detector fore optics transmission; and (7) the detector efficiency.

The OS has three modes of operation: the ion (OSI), neutral beam (OSNB), and neutral thermal (OSNT) modes. In the OSI and OSNB modes the OS is used to analyze the ram flux (or molecular beam) of ambient ions and neutrals entering the OS at spacecraft speed, respectively. We do not address OSI and OSNB extraction of ion and neutral densities and velocities in this paper: for more details see Mandt et al. (2012) and Waite et al. (2004). Here 


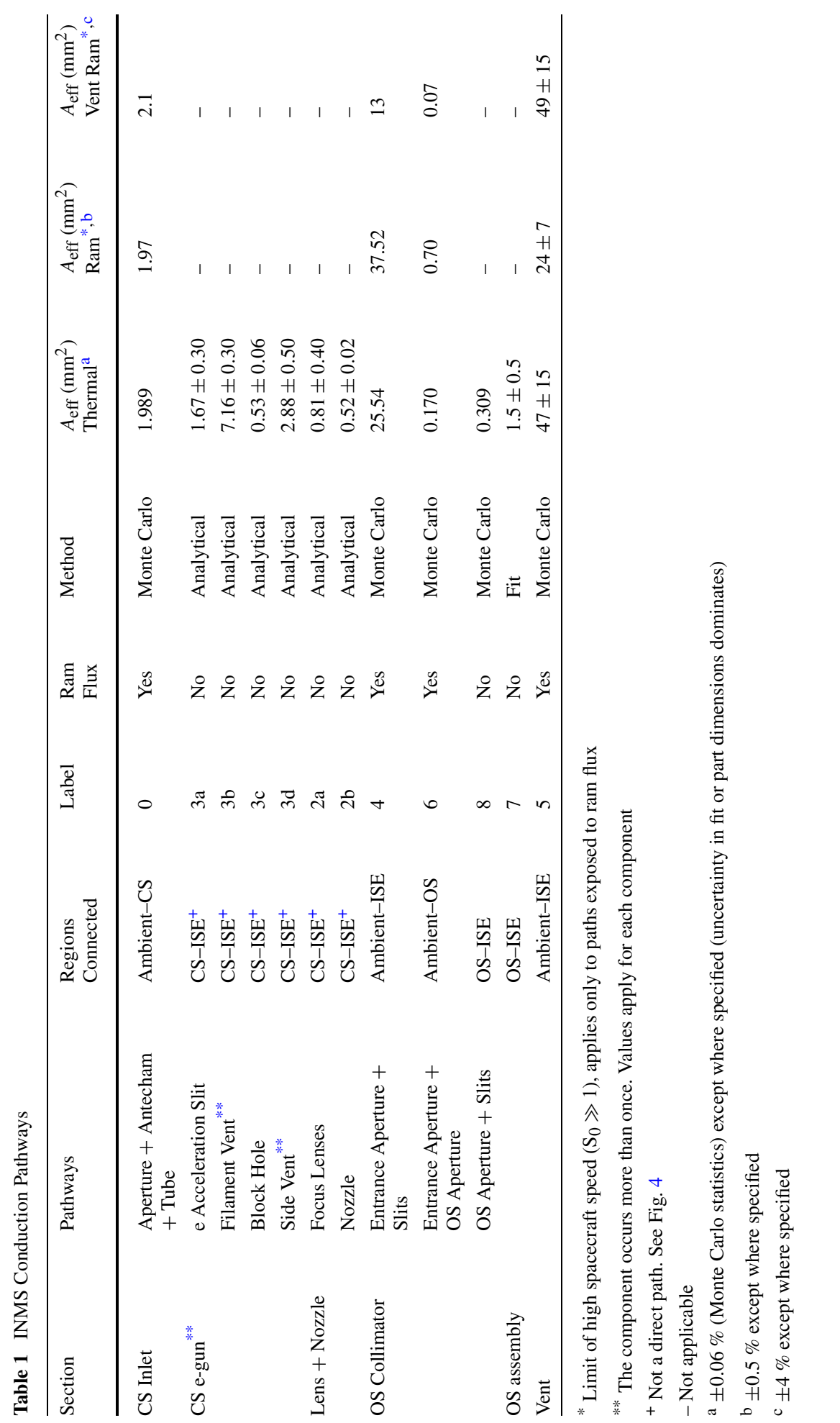


Fig. 4 (a) Flow diagram illustrating the INMS gas conductance pathways, effective areas, and role of the ram factor model parameters $F, F^{\prime}, S_{0}, \theta$, $\phi$. Pathways are labeled according to Table 1 , and colored as in Fig. 3. Dashed boxes: pathways (CS inlet, OS collimator, vent) for which effective areas were estimated by Monte Carlo simulations. Dotted box (OS assembly): effective area estimated by fit to Cassini T85 \& T88 data. Bold Boxes: effective areas estimated analytically. (b) Detail on the conductance pathways comprising each of the two CS e-gun assemblies indicated in (a) and considered in Eq. (A.7). We estimated the effective areas of all CS e-gun pathways analytically
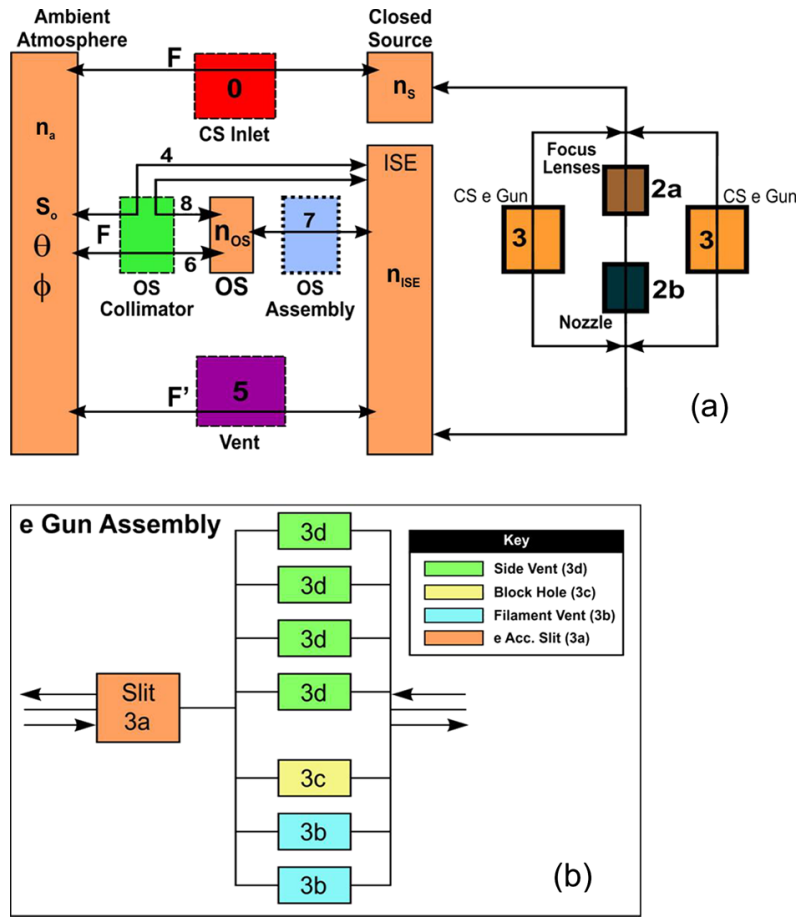

we restrict our analysis of the OS to its significance as a neutral gas conduction pathway to/from the ambient environment, and the effect on the neutral gas densities in both sources. While the instrument sensitivity correction factor discussed in Sect. 4 applies equally to all four INMS modes (CS, OSI, OSNB, OSNT), the new ram factor modeling only affects the sensitivities of the CS and OSNT modes. The OSNT mode measures the thermalized neutral gas in the OS (Waite et al. 2004), i.e., residual neutral gas molecules which have already undergone collisions in the instrument (contrary to OSNB mode which analyzes the ram neutral beam that has not undergone wall collisions). The OSNT mode thereby corresponds to operating the OS in a manner analogous to the CS. While OSNT mode is very rarely used, we discuss the OSNT sensitivity in this paper because data acquired in this mode during the T85, T88 and T96 flybys is relevant for our analysis (Appendix B).

\section{The New INMS Sensitivity Model: In Summary}

We have re-analysed the ram factor and detector gain of the Cassini's spacecraft's Ion Neutral Mass Spectrometer, and have identified an effect of (1) gas leakage pathways out of the instrument, and (2) a reduction of the overall instrument calibration sensitivity $s_{f}$ factors, which largely explains previous systematic differences between the Titan atmospheric neutral and ion density measurements of INMS and other Cassini systems. We carried out the ram factor modelling by solving the gas flow equations through the INMS, considering the gas entry and exit pathways, and internal instrument gas conduction pathways as detailed in Table 1, Figs. 3 and 4, and Appendix A. Since the ram flux arriving at the instrument has a non-isotropic molecular velocity distribution, we performed Monte Carlo simulations 
Table 2 Ram Factor Empirical Fitting Constant Values

\begin{tabular}{llllllllllllll}
\hline $\mathrm{Q}_{1}$ & 0.368 & $\mathrm{Q}_{6}$ & 0.933 & $\mathrm{Q}_{11}$ & 0.021 & $\mathrm{G}_{4}$ & 1.102 & $\mathrm{H}_{4}$ & 0.315 & $\mathrm{H}_{9}$ & 0.210 & $\mathrm{M}_{1}$ & $0.560 \mathrm{~mm}^{2}$ \\
$\mathrm{Q}_{2}$ & 0.648 & $\mathrm{Q}_{7}$ & 0.742 & $\mathrm{Q}_{12}$ & 1.787 & $\mathrm{G}_{5}$ & 0.200 & $\mathrm{H}_{5}$ & 1.143 & $\mathrm{H}_{10}$ & 0.435 & $\mathrm{M}_{2}$ & 0.150 \\
$\mathrm{Q}_{3}$ & 0.670 & $\mathrm{Q}_{8}$ & 0.261 & $\mathrm{G}_{1}$ & 0.191 & $\mathrm{H}_{1}$ & 1.047 & $\mathrm{H}_{6}$ & 0.170 & $\mathrm{H}_{11}$ & 0.100 & $\mathrm{M}_{3}$ & 30.00 \\
$\mathrm{Q}_{4}$ & 1.876 & $\mathrm{Q}_{9}$ & 44.92 & $\mathrm{G}_{2}$ & 0.336 & $\mathrm{H}_{2}$ & 0.300 & $\mathrm{H}_{7}$ & 0.300 & $\mathrm{H}_{12}$ & 0.150 & $\mathrm{M}_{4}$ & $0.055 \mathrm{~mm}^{2}$ \\
$\mathrm{Q}_{5}$ & 0.0095 & $\mathrm{Q}_{10}$ & 43.84 & $\mathrm{G}_{3}$ & 0.396 & $\mathrm{H}_{3}$ & 0.220 & $\mathrm{H}_{8}$ & 1.461 & $\mathrm{H}_{13}$ & 0.900 & $\mathrm{M}_{5}$ & $0.098 \mathrm{~mm}^{2}$ \\
\hline
\end{tabular}

(Appendix F) versus spacecraft speed and pointing to estimate gas transmission into the instrument through individual conduction pathways in terms of the 'effective areas' of these pathways (Appendix A). With two exceptions (Table 1), we estimated the effective areas of internal pathways (i.e., not exposed to the ram flux) with standard shape-dependent analytical expressions (Dushman and Lafferty 1962) that assume an isotropic 'thermalized' velocity distribution. Since the gas flow time constants are milliseconds for volatile species (Teolis et al. 2010), we solved the steady-state problem, with a set of flow balance equations between the CS, OS, ISE and the ambient environment (Appendix A). We obtain a new spacecraft speed and pointing dependent expression for the ambient neutral gas densities $n_{a f}$ encountered by the spacecraft, in terms of the count rate $X_{f}$ for each species measured in CSN mode (see also Eqs. (A.18) and (A.22a), (A.22b)):

$$
\begin{aligned}
n_{a f} & =\frac{1}{D_{s f}}\left(\frac{T_{0}}{T_{a}}\right)^{\frac{1}{2}} \frac{X_{f}}{s_{f}}, \\
D_{s} & =\left(R_{0}+R_{2} R_{1}\right) F+R_{3} R_{1} F^{\prime},
\end{aligned}
$$

where $F$ and $F^{\prime}$ are given in terms of spacecraft speed and pointing by Eqs. (A.13) and (A.16), $T_{0}$ is room temperature $(293 \mathrm{~K}), R_{1}$ is a constant 0.6254 , and the estimated (speed and pointing-dependent) $R_{0}, R_{2}$ and $R_{3}$ factors can be (i) obtained from lookup tables (to be made available on the PDS, pending publication of this work), or (ii) approximated by the empirical fitting functions given in Appendix $\mathrm{C}$ with the parameter values in Table 2. Speed and pointing are expressed in terms of the speed ratio $S_{0}$ (Eq. (A.14)) and the ram $(\theta)$ and azimuthal $(\varphi)$ angles shown in Fig. 3. We calibrated the model against the T85 and T88 Titan flybys during which the spacecraft pointing swept over a wide range of angles (ideal for constraining the model) aiming toward, and then away from the vent (Appendix B). During the inbound portion of these two flybys the INMS operates 'in reverse' as discussed in Appendix B, with the ram flux entering the instrument through the vent, and escaping via the CS and OS. In Fig. 5 we show the pointing dependence of the CS ram factor $D_{s}$ with respect to the spacecraft surfaces in view, and in Fig. 6 we give the range of pointing directions not shadowed by the spacecraft (Appendix D) where the model is applicable. The model is valid in the 'collisionless' regime below $\sim 4 \times 10^{-9} \mathrm{~kg} / \mathrm{m}^{3}\left(\sim 8 \times 10^{16}\right.$ molecules $\left./ \mathrm{m}^{3}\right)$ for which the molecular mean free path in all directions (including the ram direction) exceeds the spacecraft size, such that the ram enhancement around the spacecraft is insufficient to scatter significant flux toward or away from the INMS. To date this density limit has not been exceeded during any Titan encounters (Figs. 7, 8 and 9).

In addition to the revised ram factor modeling, the species-dependent sensitivity factors $s_{f}$ used to date, must be lowered by a (species-independent) correction factor $\beta=1.55 \pm$ $21 \%$ (again, with the revised values to be made available on the PDS) as explained in Sect. 4 . The revised $s_{f}$ values apply equally to the neutral (i.e., the CSN and OSNT modes) and the ion densities (i.e., the OSI and OSNB modes). 


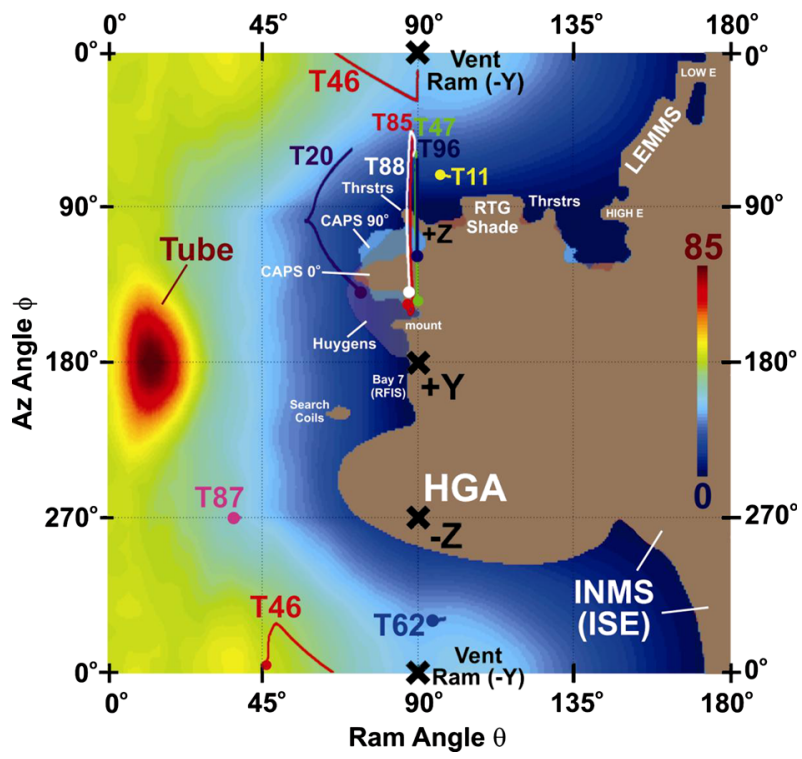

Fig. 5 Color: The estimated CSN ram factor $D_{s}$ for $S_{0}=20$, vs. $\theta$ and $\varphi$. Sensitivity enhancements can be seen for ram pointing into (i) the antechamber-to-CS tube and (ii) the vent. Brown shaded region: ram directions shadowed by the Cassini spacecraft with 0 degrees CAPS angle. The cases of 90 degrees CAPS angle, and the Huygens probe, are shown in light blue and purple shades, respectively. Here $D_{s}$ does not include the effect of the spacecraft, which is only important for ram directions approaching within a few degrees of the shadow zone (Fig. 6). Lines show the ram direction traces from -400 to $+400 \mathrm{sec}$ from closest approach, for the flybys shown in Figs. 12, 13 and 19 (the flybys all had $\mathrm{S}_{0}$ close to 20). Each trace terminates at the dot $(+400 \mathrm{sec})$

Fig. 6 Range of pointing directions outside the spacecraft shadow zone (blue) where our revised INMS ram factor modeling is applicable. Contours show the cutoff for $S_{0}=5,10$, 20 and 50, within which more than $5 \%$ of the ram flux is shadowed by the spacecraft. With increasing $S_{0}$ the contours get closer to the shadow zone edge as the ram flux thermal angular spread narrows (in the spacecraft reference frame) with increasing spacecraft speed

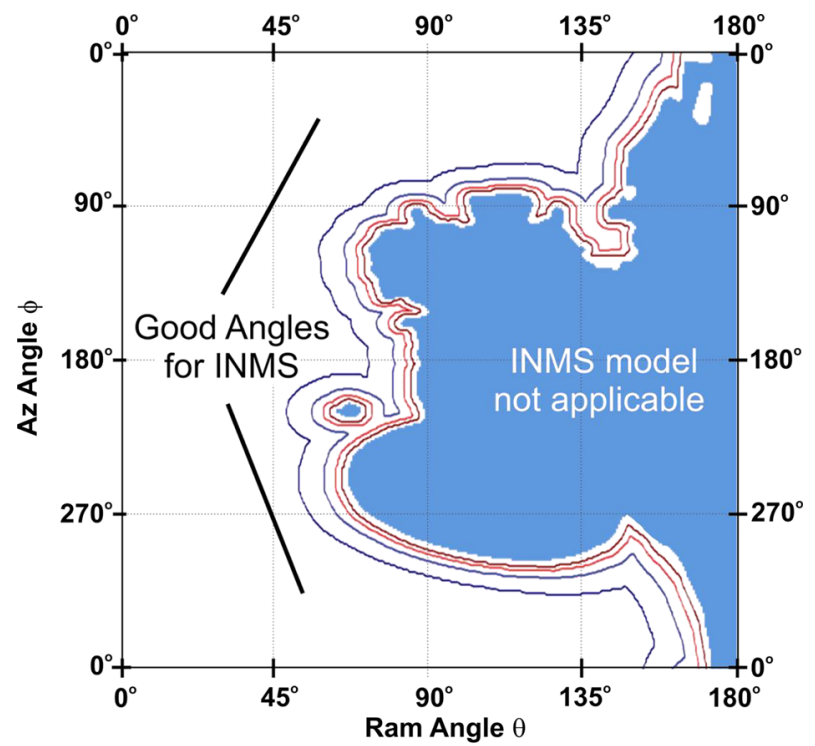




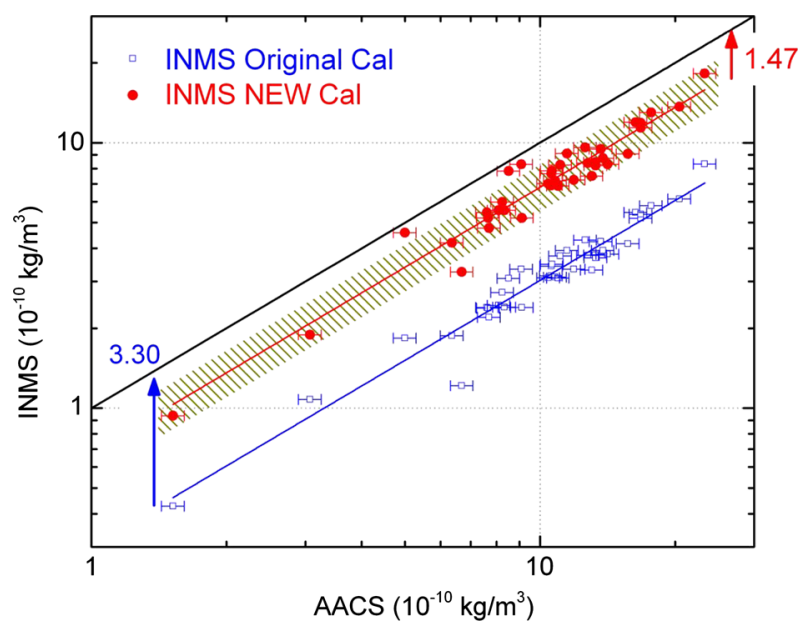

Fig. 7 Comparison of INMS and AACS Titan closest approach densities for multiple flybys, and the INMS/AACS ratio fit, with the original and new INMS calibration models. Error bars show the AACS $\pm 5.8 \%$ (Feldman et al. 2007) $1 \sigma$ random (measurement) error. The INMS random errors bars in the range $0.02-0.1 \%$ are not visible on this scale. The error of the ratio is dominated by the systematic (modeling) error of both systems. The INMS systematic error of $\pm 23 \%$ (i.e., the range by which all the points could be plausibly shifted vertically together) is shown by the hashed region to distinguish from random error. The hashed region does not encompass the 1.0 ratio line, and therefore the INMS systematic error is insufficient by itself to account for the 1.47 ratio with AACS

Fig. 8 Same as Fig. 7 but comparing INMS and NAV. The INMS systematic error of $\pm 23 \%$ encompasses the 1.0 ratio line, and is therefore sufficient to account for the 1.16 ratio with NAV irrespective of the (as yet undetermined) NAV systematic error

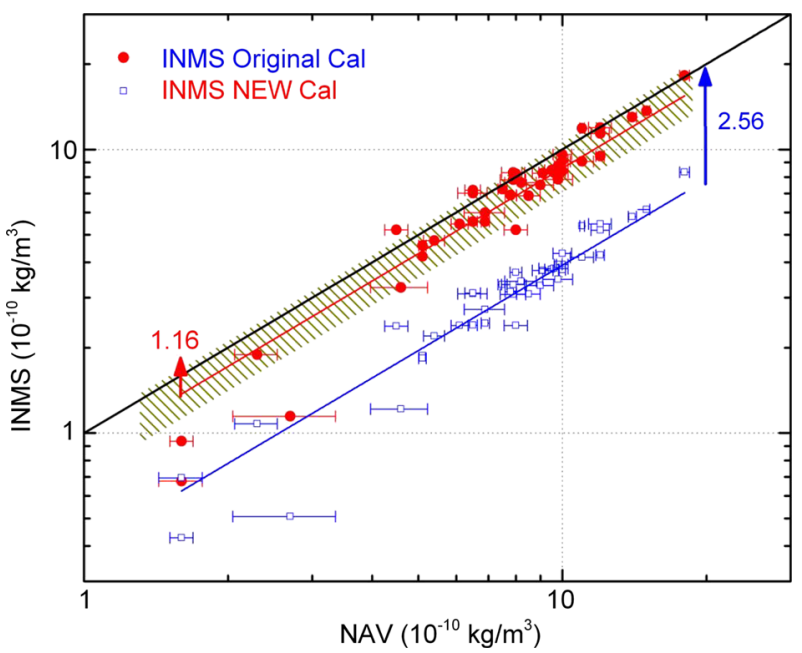

The original INMS calibration model has assumed as the basis for estimating the ram factor, an 'ideal' closed source with no leakage pathways other than the CS inlet (Fig. 3), resulting in an under-estimation of the ambient gas density required to pressurize the closed source. Contrary to the original model, we find here (Eq. (A.7)) that the total effective area of the leakage paths out of the CS $\left(3.32 \mathrm{~mm}^{2}\right)$ actually exceeds that of the CS inlet $\left(\sim 2 \mathrm{~mm}^{2}\right)$ as shown in Table 1. After escaping the CS, the vent serves as the primary (though not the only) means of escape of the gas into space. 
Fig. 9 Same as Figs. 7 and 8 but comparing NAV and AACS, showing the small $27 \%$ difference of densities measured by these two systems

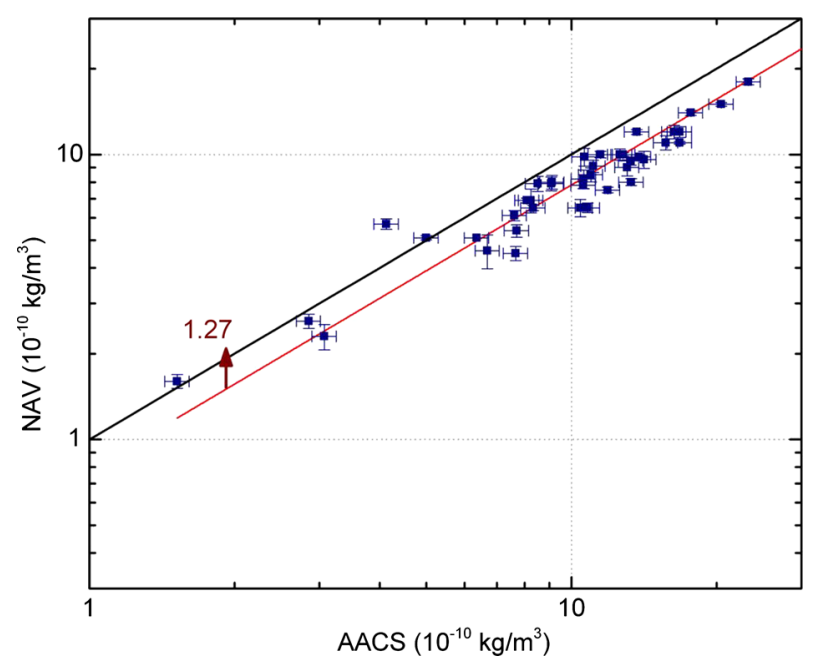

Together, these considerations yield a spacecraft pointing and speed dependent correction factor for the (CSN) neutral densities (see also Eq. (E.5))

$$
\text { correction factor }=\beta \frac{F}{D_{s}}=\beta \frac{F}{\left(R_{0}+R_{2} R_{1}\right) F+R_{3} R_{1} F^{\prime}}
$$

to the original INMS calibration model (Fig. 10). In the special case of zero degrees INMS ram angle pointing (assuming suprathermal spacecraft speeds, as is typical), the CSN correction factor is approximately a constant: $\sim 2.2 \pm 23 \%$ (Appendix E) and approximately independent of the gas species. These corrections supersede the factor three used by several previous INMS based studies (in anticipation of the more detailed determination reported here).

\section{Correction to the Calibration Sensitivity Factors $s_{f}$}

The calibration sensitivity factors for $\mathrm{CH}_{4}$ and $\mathrm{N}_{2}$ were measured pre-launch by exposing the INMS to gas leaked into a laboratory calibration chamber, and comparing (1) the instrument count rate for each species, to (2) the ambient gas density measurement from a separate chamber-mounted pressure sensor. Following a subsequent pre-launch characterization testing phase [detailed by Waite et al. 2004] the decision was made to operate the INMS primary (high-sensitivity) secondary electron multiplier (SEM) detector at $2701 \mathrm{~V}$, rather than the $2500 \mathrm{~V}$ initially used for calibration. The voltage increase resulted in an increase of SEM gain, which was estimated at the time to increase the sensitivity by a factor of 1.27. Accordingly, the $s_{f}$ factors for multiple different gas species, measured post-launch in the laboratory, with the INMS refurbished engineering unit, or REU, have been scaled to the original $\mathrm{CH}_{4}$ and $\mathrm{N}_{2}$ flight unit calibration, times 1.27.

However the characterization tests also subjected the SEM to sufficient counts to degrade its performance, by lowering the gain as shown in Waite et al. (2004). The detector gain has been estimated during pre-launch testing, and in flight, by fitting (by linear regression) the proportionality constant of the SEM anode current to the count rate registered by the pulse counter circuitry. As part of this work, we have re-investigated the measurements performed during the characterization period, and discovered that the gain reduction was, erroneously, 
Fig. 10 Correction factor to densities estimated from the original calibration model, vs. ram and azimuthal angle. The correction has an uncertainty of $\pm 23 \%$. In this example $S_{0}=20$ typical of a Titan flyby, i.e. $m=28 \mathrm{u}$ per charge for $\mathrm{N}_{2}$, $u=6 \mathrm{~km} / \mathrm{s}, T_{a}=150 \mathrm{~K}$ (Eq. (A.14)). Near $\theta=15 \mathrm{deg}$, $\varphi=180 \mathrm{deg}$ the ram flux is aimed into the antechamberto-CS tube, resulting in a high sensitivity spot. At higher $\theta$ the factor is highly $\varphi$ dependent due to the effect of the vent. Blue shaded region at right is the spacecraft shadowing zone with CAPS at $90 \mathrm{deg}$. Note at $0^{\circ} \mathrm{ram}$ that the factor is $\sim 2.2$. (a) Using Eq. (E.5). The 'noise' in the contour lines originates from statistical error in effective areas estimated by Monte Carlo simulations. (b) Correction factor resulting from the

Eqs. (C.1a)-(C.4j) empirical fitting functions
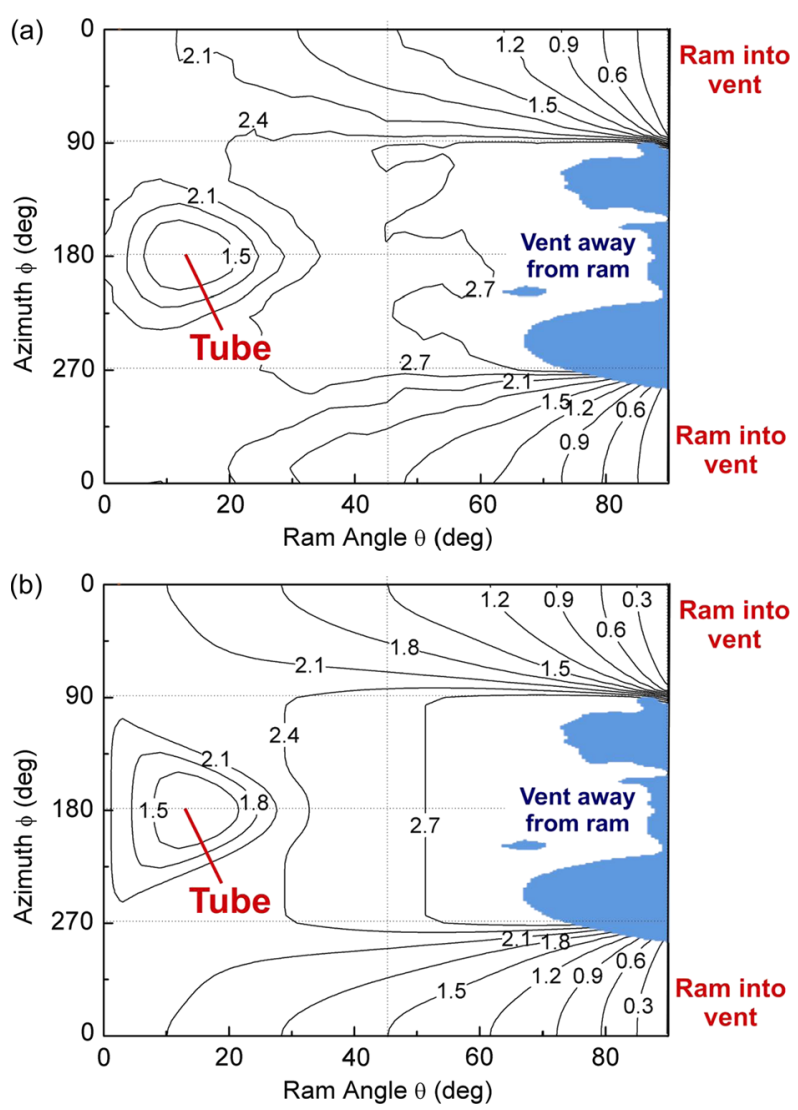

not included in the 1.27 sensitivity adjustment factor. Additionally, the factor 1.27 itself appears to have over-estimated the effect of the SEM voltage change because only two test runs were compared (at the end of the characterization period), leading to a large margin of error. As shown in Fig. 11a [based on the data shown in Waite et al. 2004], the pre-launch characterization tests produced a gain decrease of $\sim 82 \%$ from a starting value of $\sim 7 \times 10^{7}$ over the 77 test runs performed, even after accounting for the SEM voltage change from 2500 to 2701 V. Fortunately most of the voltage pulses (produced by ion strikes on the SEM) are still sufficiently in excess of the discriminator threshold to be registered by the pulse counter, despite the gain loss, and therefore the effect of the gain loss on the count rate is not very drastic. In Fig. 11b we plot the count rate versus gain from the characterization tests: a linear fit (on logarithmic scale) yields:

$$
s_{f} \propto \operatorname{Gain}^{0.096}
$$

over the relevant gain range, with the non-zero exponent a consequence of the finite pulse height distribution width. By Eq. (4), an $82 \%$ gain loss translates to a factor 1.21 count rate reduction during the characterization tests. Since the species calibration sensitivity factors $s_{f}$ used to date have assumed a factor 1.27 increase, rather than a reduction, we find here that these sensitivity factors should be reduced by $1.21 \pm 21 \%$ times 1.27 , i.e., $\beta=1.55 \pm 21 \%$. Gain measurements on the flight unit during the T23, 25, 29, 40, 41, 49, 51, 55, 77, 84, and 98 Titan flybys reveal no statistically discernible trend, implying that $\beta$ has remained 
Fig. 11 (a) SEM gain versus INMS test run number, from the 77 pre-launch characterization tests, showing the loss of gain for three SEM voltages: 2500 (blue), 2701 (red, standard value used in flight) and $2977 \mathrm{~V}$ (green). (b) Count rate (relative units) versus gain from the same test runs. Despite the $\sim 82 \%$ gain loss, the weak dependence of count rate on gain yields a sensitivity reduction factor of only $1.21 \pm 21 \%$
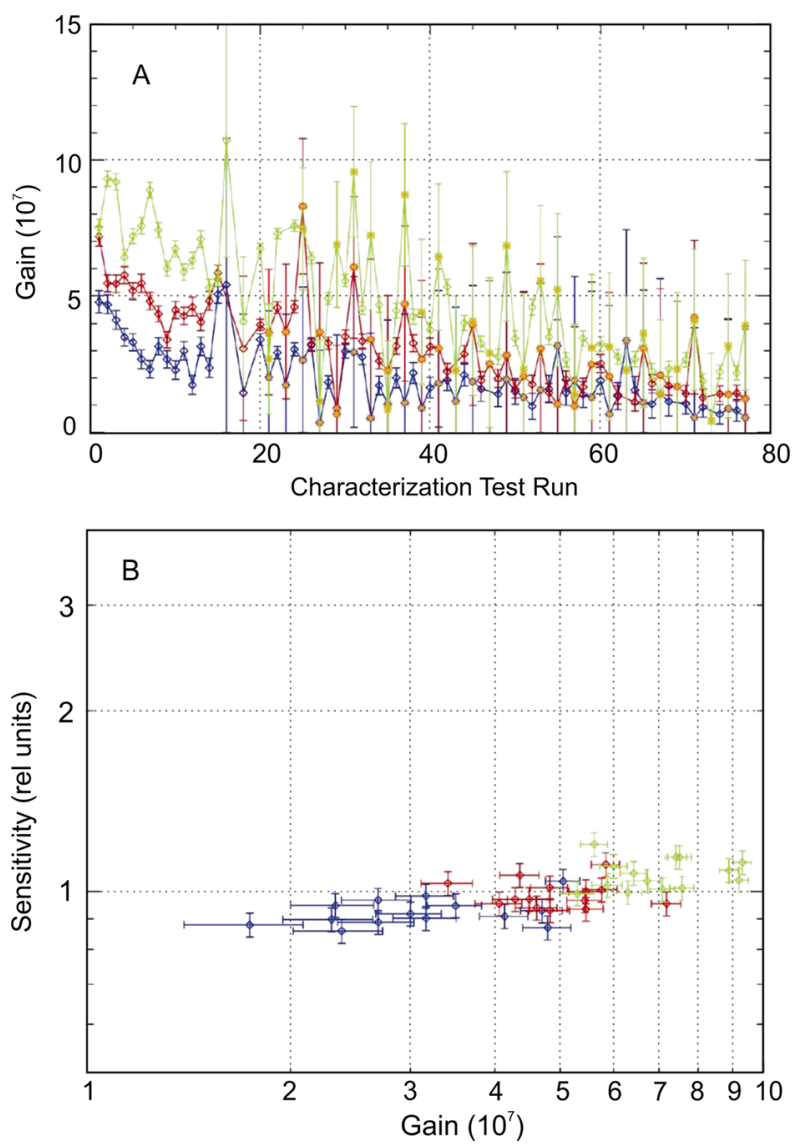

approximately unchanged since launch. The INMS team plans to update the factors on the Planetary Data System, pending publication of the results reported here.

\section{Explaining ‘anomalous' Titan Flybys}

The ram factor modeling also provides an explanation for the T11, 20, 46, 47, 62, 85, 88 and 96 Titan encounters, where the original calibration [Appendix E] (1) over-estimates Titan's $\mathrm{N}_{2}$ density (sometimes drastically), and/or (2) yields an unreasonably large difference between the inbound and outbound measured densities. As shown in Fig. 5, all of the relevant encounters had spacecraft pointing far off ram, and in all cases the vent ram flux (not included in the original calibration model) played a major role in the signal. As shown in Fig. 12 for T11, 20, 46, and 62 (and Fig. 13 for the T47, 85, 88, and 96 flybys discussed in Appendix B), the new ram factor modelling largely corrects the problems, with (aside for spacecraft shadowing effects) the only uncorrected discrepancy being observed at T46 where the inbound/outbound difference appears to be real. Hence the new ram factor modelling not only increases the accuracy of the INMS neutral densities estimates, but also expands the list of Titan encounters from which reliable densities may be extracted. 


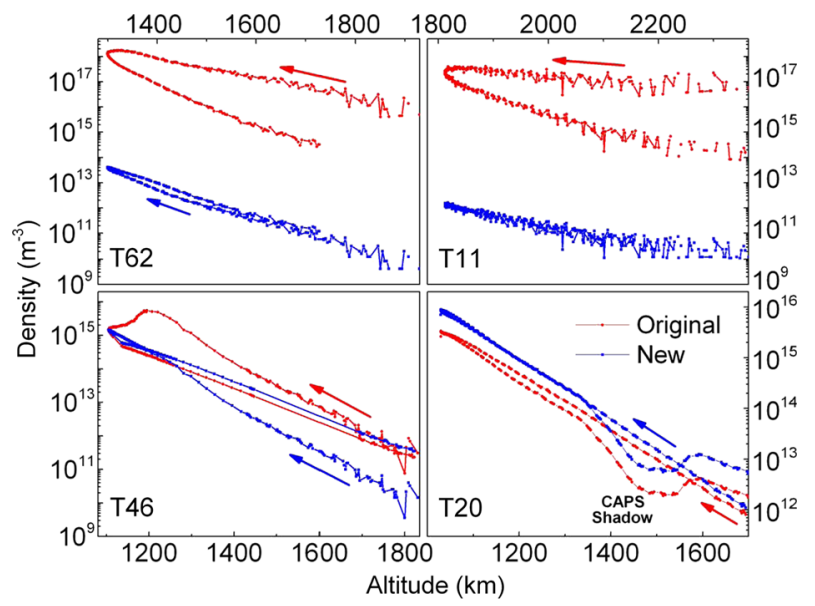

Fig. 12 Estimated $\mathrm{N}_{2}$ density vs. altitude from the mass 28 channel for the Cassini T11, 20, 46, and 62 Titan encounters between -400 and $400 \mathrm{sec}$ from CA, using the original 'ideal' calibration (red circles), and the new ram factor model (blue squares) with $s_{f}=4.05 \times 10^{-10} \mathrm{~m}^{3} / \mathrm{s}$ for $\mathrm{N}_{2}$ (the factor $\beta$ is applied to $s_{f}$ ). The arrows indicate the inbound branch of the data. The original calibration yields an inbound/outbound density difference as the instrument pointing changed over the flybys (Fig. 5). However the new model anticipates and largely cancels the effect. High-altitude outbound data (not shown) over-estimate the densities due a significant contribution from residual gas in the instrument. On the outbound portion of T20 the ram pointing is briefly shadowed by the tip of CAPS ELS (at 0 deg rotation angle) as can be seen in Fig. 5, resulting in a brief dip in signal which is uncorrected by the new model. Density measurements in the dip are therefore not valid and are shown only to illustrate the effect. The T11 and 62 flyby pointing (nearly steady through the flyby) was greater than $90 \mathrm{deg}$ ram but close to vent ram. Hence the original calibration drastically under-estimates the instrument sensitivity, and thereby over-estimates the T11 and 62 densities. The new model gives much lower, more reasonable densities

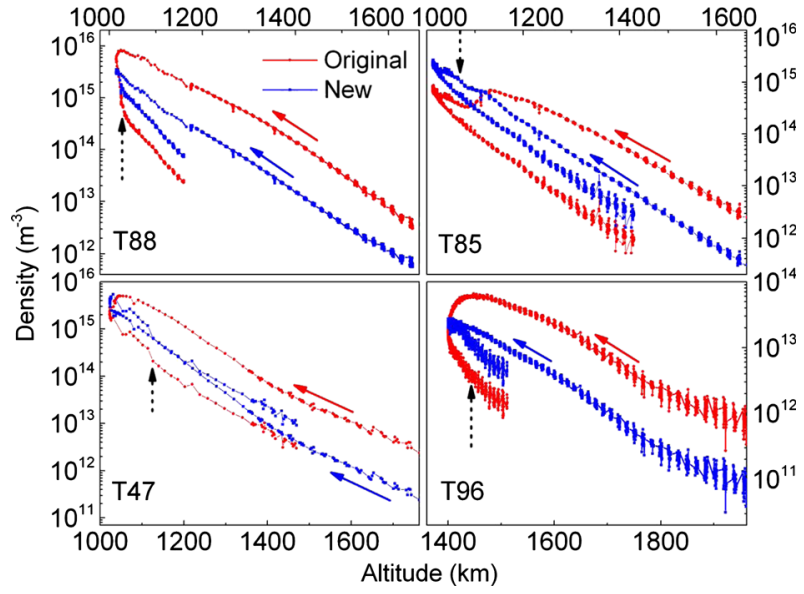

Fig. 13 Same as Fig. 12 for the T47, 85, 88 and 96 flybys. These four flybys had similar geometry (Fig. 5), i.e., near $90 \mathrm{deg}$ ram angle, with vent ram angle sweeping through 90 degrees. The original calibration yields an unreasonable inbound/outbound difference in the estimated density, with a sudden drop near closest approach as the vent ram angle exceeds 90 degrees. The new model anticipates and cancels the effect to a large degree. However the new model does not include spacecraft shadowing, and therefore somewhat under-estimates the density after (dashed arrow) the spacecraft obstructs the ram view direction of INMS (particularly for T85, 88 and 96 since CAPS was at 90 deg during those flybys, Fig. 20). Hence density measurements after the dashed arrow are not valid 


\section{Comparison of INMS Densities to Other Cassini Systems}

AACS and NAV each use different methods to estimate the spacecraft drag acceleration $\vec{a}_{A t m}$, related to the gas mass density $\rho$ by

$$
\overrightarrow{\mathrm{a}}_{\mathrm{Atm}}=-\frac{\rho \mathrm{V}^{2} \mathrm{CA}}{2 M} \hat{\mathrm{V}}
$$

or,

$$
\rho=\frac{2 M\left|\overrightarrow{\mathrm{a}}_{\mathrm{Atm}}\right|}{\mathrm{CAV}^{2}},
$$

where $M$ denotes the spacecraft mass, $\mathrm{C}$ the drag coefficient, $\overrightarrow{\mathrm{V}}=\mathrm{V} \hat{\mathrm{V}}$ the spacecraft velocity in the gas reference frame, and A the spacecraft cross section area projected along $\vec{V}$. C can be estimated using formulae given in Stalder and Zurick (1951). We use $\mathrm{C}=2.1 \pm 0.1$, which is the value calculated for a cylinder in free molecular flow, and also that found in Monte Carlo simulations of the gas flow interaction with the Cassini spacecraft (Liechty 2006). This is a reasonable drag coefficient value when compared with results determined using orbital data of Earth-orbiting satellites (Moe and Bowman 2005).

AACS measures the acceleration in two ways: (1) directly through an accelerometer (powered on only for selected Titan flybys), or (2) through the atmospheric drag torque $\overrightarrow{\mathrm{T}}_{\mathrm{Atm}}$ on the spacecraft, determined by way of the torques and duty cycles of the thrusters (most flybys) which fire automatically to stabilize the spacecraft orientation. $\overrightarrow{\mathrm{T}}_{\text {Atm }}$ satisfies the spacecraft equation of rotational motion (Lee 1999; Lee and Hanover 2005):

$$
\overleftrightarrow{\mathrm{I}}_{\mathrm{SC}} \dot{\vec{\omega}}+\vec{\omega} \times\left(\overleftrightarrow{\mathrm{I}}_{\mathrm{SC}} \vec{\omega}+\overrightarrow{\mathrm{H}}_{\mathrm{RWA}}\right)=\overrightarrow{\mathrm{T}}_{\mathrm{Thrstr}}+\overrightarrow{\mathrm{T}}_{\mathrm{Atm}}+\overrightarrow{\mathrm{T}}_{\mathrm{RWA}}+\vec{\varepsilon}
$$

or,

$$
\overrightarrow{\mathrm{T}}_{\mathrm{Atm}}=\overleftrightarrow{\mathrm{I}}_{\mathrm{SC}} \dot{\vec{\omega}}+\vec{\omega} \times\left(\overleftrightarrow{\mathrm{I}}_{\mathrm{SC}} \vec{\omega}+\overrightarrow{\mathrm{H}}_{\mathrm{RWA}}\right)-\overrightarrow{\mathrm{T}}_{\mathrm{Thrstr}}-\overrightarrow{\mathrm{T}}_{\mathrm{RWA}}-\vec{\varepsilon}
$$

where $\stackrel{\leftrightarrow}{\mathrm{I}}_{\mathrm{SC}}$ denotes the spacecraft's inertia tensor, $\vec{\omega}$ the spacecraft's angular velocity, $\dot{\vec{\omega}}$ the spacecraft's angular acceleration, $\overrightarrow{\mathrm{T}}_{\mathrm{RWA}}$ and $\overrightarrow{\mathrm{H}}_{\mathrm{RWA}}$ the torque and angular momentum of the reaction wheels (zero if the wheels are powered off), and $\overrightarrow{\mathrm{T}}_{\text {Thrstr }}$ the torque exerted by the thrusters. The spacecraft's inertia tensor is estimated by ground software, and has previously been confirmed by an in-flight calibration technique (Lee and Wertz 2002). An onboard attitude estimator (the Kalman-Bucy filter) provides $\vec{\omega}$ at $125 \mathrm{~ms}$ time intervals. $\overrightarrow{\mathrm{T}}_{\text {Thrstr }}$ is extrapolated by flight software from the estimated force impulse due to all thruster firings, including effects due to the thruster rise and tail-off dynamics. The impulses are converted into the per-axis torque impulses using the known moment arms of all the thrusters. $\vec{\varepsilon}$ contains the torques from solar radiation, Titan's gravity gradient, etc., but these torques are negligible $(<1.1 \mathrm{mNm})$ to first order (Lee and Hanover 2005). The drag acceleration is related to the atmospheric torque by (Feldman et al. 2007; Lee and Hanover 2005; Sarani 2007):

$$
\overrightarrow{\mathrm{T}}_{\mathrm{Atm}}=-M \overrightarrow{\mathrm{a}}_{\mathrm{Atm}} \times \overrightarrow{\mathrm{r}}_{\perp}
$$

or,

$$
\overrightarrow{\mathrm{a}}_{\mathrm{Atm}}=\frac{\overrightarrow{\mathrm{T}}_{\mathrm{Atm}} \times \overrightarrow{\mathrm{r}}_{\perp}}{M \mathrm{r}_{\perp}^{2}},
$$




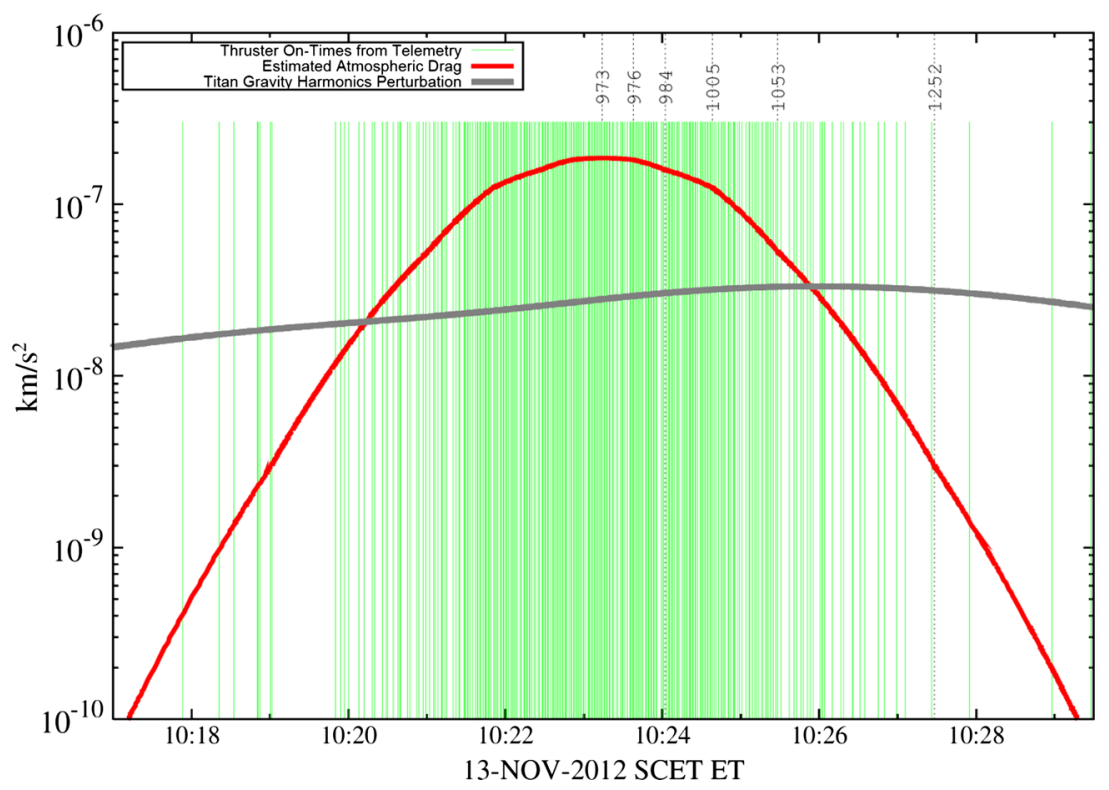

Fig. 14 T87 NAV results. Green spikes: thruster impulses estimated from AACS and used as input to the NAV model. Grey Line: gravity harmonic perturbation model. Red Line: atmospheric drag from the Eq. (9b) fit to the NAV data, with vertical dotted lines indicating the layer center times and altitudes in $\mathrm{km}$ (outbound shown, same altitudes inbound)

where $\vec{r}=\vec{r}_{C P}-\vec{r}_{C M}$ and $\vec{r}_{\perp}=\vec{r}-(\vec{r} \cdot \hat{V}) \hat{V}$, with $\vec{r}_{C P}$ and $\vec{r}_{C M}$ the positions of the spacecraft centers of pressure and mass as estimated by ground software (Feldman et al. 2007; Lee and Hanover 2005; Lee and Lim 2013; Sarani 2007, 2009). We therefore obtain (by substituting Eq. (6b) into (7b), then (7b) into (5b)) the density in terms of thruster torque:

$$
\rho \approx \frac{2}{\mathrm{CAV}^{2}} \frac{\left|\left(\stackrel{\leftrightarrow}{\mathrm{I}}_{\mathrm{SC}} \dot{\vec{\omega}}+\vec{\omega} \times\left(\stackrel{\mathrm{I}}{\mathrm{SC}}_{\vec{\omega}} \overrightarrow{\mathrm{H}_{\mathrm{RWA}}}\right)-\overrightarrow{\mathrm{T}}_{\mathrm{Thrstr}}-\overrightarrow{\mathrm{T}}_{\mathrm{RWA}}\right) \times \overrightarrow{\mathrm{r}}_{\perp}\right|}{\mathrm{r}_{\perp}^{2}}
$$

where we have dropped the negligible $\vec{\varepsilon}$ term. Similar methodologies have provided atmospheric density estimates of Venus (Espiritus and Tolson 1995) and Mars (Spencer et al. 1999), and of Titan using the Huygens HASI accelerometer data (Fulchignoni 2007; Fulchignoni et al. 2005; Zarnecki et al. 2003).

NAV uses radiometric Doppler tracking data to estimate the spacecraft velocity, which requires HGA pointing and transmission to earth at the time of the measurement. All the forces acting on the spacecraft are modeled (such as thruster impulses, gravitational harmonics and atmospheric drag, see Fig. 14) and integrated with the equations of motion along the computed spacecraft trajectory. The calculation is iterated to fit the model parameters to the tracking data by a least squares method. Using this approach a model of the atmospheric density (an exponential profile vs altitude) is fit to yield the drag acceleration (via Eq. (5a)) implied by the tracking data. The total one-sigma (random) error of the result (typically a few percent) includes uncertainty in $\mathrm{C}( \pm 1.6 \%), \overrightarrow{\mathrm{V}}( \pm 0.005 \%), \mathrm{A}( \pm 0.7 \%)$, $M( \pm 0.73 \%)$, and the Doppler-based measurement and estimation error (varies between flybys, of order $\pm 1 \%$ ). Opportunities for earthward HGA pointing are scarce due to the 
pointing requirements of other spacecraft systems. Therefore, apart from the T70, T87, and T107 encounters, every low altitude Titan flyby of the Cassini mission is "in the blind", as far as NAV is concerned, meaning that no tracking data is captured during the time Cassini dips into the atmosphere and experiences drag. All NAV can do in these cases is to measure (after the flyby) the net acceleration of drag.

Only the T70, T87 and T107 flybys had continuous HGA pointing to earth, yielding altitude resolution in the Doppler data. To retrieve the neutral mass density during these flybys, the model atmosphere is divided into isothermal altitude layers between 950 and $1300 \mathrm{~km}$. The density and (using Eq. (5a)) the drag acceleration at altitude $h$ are modeled as

$$
\begin{aligned}
& \rho=\rho_{i} \exp \left(\frac{h_{i}-h}{H_{i}}\right) \\
& \overrightarrow{\mathrm{a}}_{\mathrm{Atm}}=-\hat{\mathrm{V}} \frac{\mathrm{V}^{2} \mathrm{CA}}{2 M} \rho_{i} \exp \left(\frac{h_{i}-h}{H_{i}}\right)
\end{aligned}
$$

where $\rho_{i}$ is the density at a reference altitude $h_{i}$ and $H_{i}$ is the scale height for the $i$ th layer of the atmosphere:

$$
H_{i}=\frac{h_{i}-h_{i+1}}{\ln \left(\rho_{i+1} / \rho_{i}\right)} .
$$

The layer thickness is defined such that the accumulated $\Delta \mathrm{V}$ from drag is $\sim 10$ times the noise level of the Doppler during Cassini's traversal of the layer. The layer placement chosen for T87 is shown in Fig. 14. Note that different layer placements were allowed between the inbound and outbound legs to capture variations in the atmosphere.

In Figs. 7-9 we compare the INMS Titan closest approach densities for multiple flybys to those of AACS and NAV, with the original and new calibration models. As shown the original calibration model yields systematic deviations of INMS from AACS and NAV, with AACS/INMS and NAV/INMS ratios of 3.30 and 2.56. However the new model yields much better agreement, with ratios of 1.47 and 1.16, respectively. Therefore INMS shows better agreement with NAV than AACS, reflecting the small factor of 1.27 disagreement between NAV and AACS (Fig. 9). The one-sigma errors on the ratios are $\pm \sqrt{\sigma_{\text {INMS }}^{2}+\sigma_{\text {AACS }}^{2}}$, $\pm \sqrt{\sigma_{\mathrm{INMS}}^{2}+\sigma_{\mathrm{NAV}}^{2}}$ and $\pm \sqrt{\sigma_{\mathrm{AACS}}^{2}+\sigma_{\mathrm{NAV}}^{2}}$, where $\sigma$ is the systematic (i.e. model-dependent) standard error of the densities. The one-sigma AACS random error is estimated to be $\pm 5.8 \%$ (Feldman et al. 2007), and those for NAV are usually a few percent shown as in Figs. 89. However, we ignore the random measurement error since it contributes negligibly due to the large number of Titan flybys used to compute the ratios. In contrast to the random error, the AACS and NAV systematic errors are presently unknown, and we therefore include only the INMS contributions in Figs. 7-9 (cross-hatched regions). As shown in Fig. 8 $\sigma_{\text {INMS }}=23 \%$ yields agreement (i.e. within error) between INMS and NAV irrespective of any (systematic) NAV error. Interestingly the INMS error by itself is not sufficient to yield agreement with AACS (Fig. 7). This remaining INMS/AACS disagreement, at least $24 \%$ outside INMS error, is currently unexplained. At present we can say only to our knowledge that the disagreement cannot be entirely attributed to uncertainty in INMS modeling and measurements.

The total electron density is measured by RPWS and is a reliable proxy for the total ion density in Titan's atmosphere between $1100-1250 \mathrm{~km}$; a region where high mass negative ions do not constitute a significant fraction of the negative charge density (Well- 
brock et al. 2013). Above $1250 \mathrm{~km}$ the RPWS density at times is higher than the INMS ion density because ion drifts at higher altitudes shift the ions velocities outside of the INMS field of view. Below $\sim 1100 \mathrm{~km}$ a significant contribution of heavy negative ions to the negative charge, and the presence of heavy positive ions above the 100 amu INMS mass range, can also effect the ratio of RPWS to INMS (Crary et al. 2009; Mandt et al. 2012; Westlake et al. 2014). Therefore, the ratio of RPWS electron densities to INMS total ion density was evaluated versus altitude between 1100 and $1250 \mathrm{~km}$ for 10 flybys at $0^{\circ} \mathrm{ram}$ angle, and found to be approximately constant in this altitude range. The profile comparison provided an average ratio of $1.37 \pm 0.6$ using the original INMS calibration model, but the ratio reduces to $0.88 \pm 0.39$ with the new model. Therefore both models yield agreement within error between INMS and RPWS, but the agreement is better with the new model. Charge balance considerations require close agreement of the ion and electron densities in the 1100-1250 km altitude range, and hence INMS and RPWS are, in effect, making independent measurements of the same quantity (i.e., ion/electron density). Accordingly, when both INMS ion and RPWS electron measurements are available and agree within error, we recommend that the ion/electron density should be evaluated as the mean value of the overlap of the INMS and RPWS error bars (ideally including random and systematic errors of both systems). CAPS-IBS also measures the ion densities. In Titan's atmosphere, the majority of the ion energy of the thermal ionosphere is kinetic energy derived as a result of the velocity of the spacecraft through the near stationary atmosphere, which allows the high energy resolution of the IBS energy spectrometer to be used as a low resolution mass spectrometer (Crary et al. 2009). The energy range of IBS overlaps with the mass range of the major components of Titan's ionosphere allowing total ion densities to be derived from IBS and compared to INMS. We compared the IBS ion densities below $100 \mathrm{amu}$ (the INMS mass range) to INMS total ion densities for 7 flybys. This comparison gives a ratio of $2.48 \pm 0.68$ with the original INMS calibration, which reduces to $1.60 \pm 0.44$ with the new calibration. We note that all errors given here are under-estimates since we have not included RPWS and IBS systematic errors.

Later in the Cassini mission the project allocated the T87 (13 Nov 2012) and T107 (10 Dec 2014) flybys during the Solstice Tour to compare the AACS and NAV densities to INMS. These two flybys were selected because the geometry allowed the first density measurements with altitude resolution to be simultaneously acquired from all three systems. The opportunity was also taken to power on the AACS accelerometer (ACC) during T87, yielding four independent density measurements versus altitude (Thrusters, ACC, NAV, and INMS). The NAV measurement required continuous HGA Earth pointing during the flyby, $\sim 52$ degrees from the ram direction. In Fig. 15 we compare the T87 densities vs altitude measured by the AACS thrusters and accelerometer, and NAV, to the INMS mass densities with the new calibration (as estimated from the $28 \mathrm{u}$ per charge $\mathrm{N}_{2}$ signal). With the exception of a partial inbound INMS-AACS disagreement, all the systems, including INMS, agree within errors on the density at all altitudes.

\section{Impact of New Calibration Model on Previous Titan Studies}

Numerous publications using INMS thermal neutral measurements include absolute densities and mixing ratios of neutral species in Titan's atmosphere and the plume of Enceladus. INMS neutral densities have primarily been used for dynamical models of Titan's atmosphere (Bell et al. 2010a, 2010b; Müller-Wodarg et al. 2008; Yelle et al. 

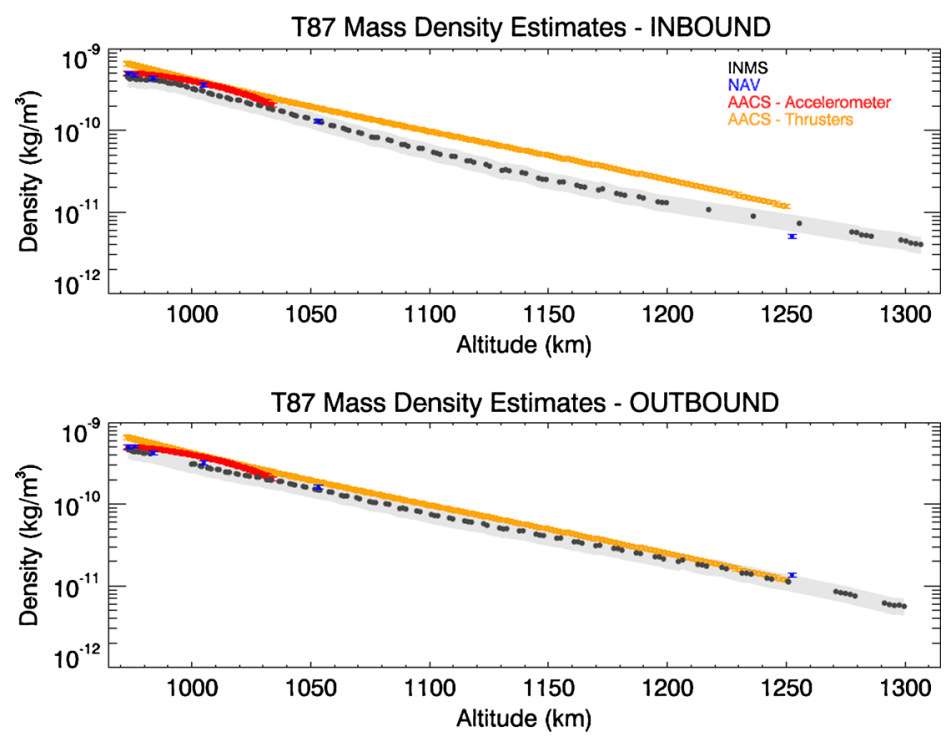

Fig. 15 T87 simultaneous density measurements by AACS-Thrusters (orange), AACS-Accelerometer (red), NAV (blue), and INMS (black) with the new calibration model. Grey shading shows INMS $\pm 23 \%$ systematic error (INMS random errors are negligible on the scale shown). T87 was the first flyby which acquired in situ measurements of the density altitude profiles by all four systems (with a repeat carried out on T107). Except for a partial inbound disagreement of AACS-Thrusters and INMS, all of the systems agree within errors

2006, 2008) and photochemical modeling (Cravens et al. 2009; Robertson et al. 2009; Vuitton et al. 2007, 2006).

Temperature derivations based on the scale height use the ratio of densities at two points in the atmosphere (Müller-Wodarg et al. 2008). For $\theta=0$ fixed flybys the temperature derivations are not affected by the (on ram) 2.2 instrument sensitivity correction constant, which cancels from the ratio. However, other publications have used the INMS density vs. altitude profiles to constrain the dynamics of the upper atmosphere (Bell et al. 2010a, 2010b; Yelle et al. 2006, 2008). Ideally, these dynamical models [e.g., Bell et al. 2010a, 2010b; Yelle et al. 2006] use densities and mixing ratios at their lower boundaries that are constrained by lower atmospheric observations from the Cassini Composite Infrared Spectrometer (CIRS) and the Huygens Atmospheric Science Investigation (HASI). For a factor 2.2 increase in INMS upper atmospheric densities these models require a warmer thermosphere to match the scaled INMS densities-assuming that these models maintain constant lower boundary conditions. These higher thermospheric densities (and simultaneously warmer temperatures) will alter key aspects of these dynamical simulations, such as the absolute value of the eddy diffusion and binary molecular diffusion coefficients at a given altitude. Some studies have found that matching the scaled up INMS densities alters the altitude of peak methane photodestruction, the implied thermal structure of the background atmosphere, and the methane vertical dynamics. These aspects in turn have been found to alter the inferred escape rates from non-hydrostatic models (Bell et al. 2010a, 2010b, 2014). These models are consistent with the kinetic treatments by Tucker and Johnson (2009) and Tucker et al. (2013) using the same scaled up INMS densities. Alternatively, some studies have matched the scaled up INMS densities but have not found differences in their inferred escape rates of methane [cf., Strobel 2010, 2012], meaning that the topic of methane escape is still a subject of active investigation and debate. 
Increasing the INMS densities by a factor of 2.2 will alter all of the major photochemical dissociation and heating rates. The optical depth calculations increase by the same factor, moving the peak of solar heating and dissociation up by almost a scale height $(\sim 70 \mathrm{~km})$. Chemical reaction rates will be modified as well, but the impact is more difficult to constrain because both production and loss rates are affected. Robertson et al. (2009) found that the peak ion densities calculated in their photochemical model shifted to a higher altitude $(\sim 60 \mathrm{~km})$ when the INMS neutral densities were increased by a factor of 2.5 , bringing their model results into better agreement with the INMS ion data. These findings have been confirmed by recent modeling (Galand et al. 2010; Richard et al. 2014; Vigren et al. 2013).

Acknowledgements The INMS team acknowledges support from NASA and the Jet Propulsion Laboratory under SwRI subcontract 1405853. Support for this work was provided by a Centre National d'Etudes Spatiales Cassini Participating Scientist grant (to VV). Titan atmospheric densities estimated by the Attitude Control team represent work done by many individuals. T. Barber, T. Burk, A. Feldman, R.S. Lim, Siamak Sarani (formerly with JPL), E.K. Wang, J.L. Webster, and Genevie Yang, our colleagues at JPL, have provided us with invaluable support. We are especially grateful to co-author and Cassini INMS Co-Investigator Hasso Niemman, who passed away July 11, 2013. Hasso leaves a decades long legacy in the field of spaceflight mass spectrometry, and his insight, analysis and detailed knowledge of the INMS were essential to this work. He will be missed by the team and the community.

Open Access This article is distributed under the terms of the Creative Commons Attribution License which permits any use, distribution, and reproduction in any medium, provided the original author(s) and the source are credited.

\section{Appendix A: Neutral Density Ram Factor Modeling}

The gas conduction pathways through the INMS are indicated in Table 1 and Figs. 3 and 4 by number-letter pairs. Ambient gas flows to the closed source (CS) via the CS inlet (subscript $0)-$ consisting of the antechamber aperture + antechamber + tube in series. As shown in Figs. 3 and 4, gas flows between the CS and the ISE through openings in the electron gun structure (3a, 3b, 3c, and 3d, see Fig. 4b), and separately (i.e. in parallel), through the focus lenses (2a) in series with the ion nozzle (2b). The ISE also communicates with the ambient environment through the OS pathways and the vent (5). The OS exchanges gas with the ambient environment through the OS collimator (6), and with the ISE by leakage (i) through the OS assembly (7), i.e., where the OS ionization region resides, or (ii) through the OS collimator (8) via slits. Ambient gas can also bypass the OS assembly by flowing directly through the collimator slits to/from the ISE (4). Gas can flow between the ISE and QMA through the ion focus lenses (Fig. 3), but this pathway is not relevant for a steady state model since there are no other gas conduction pathways out of the QMA enclosure. The conduction pathways considered in the model are summarized in Table 1 and Fig. 3, and the labeled flow diagrams in Fig. 4 show schematically how the closed source, open source and ISE densities couple to the ambient density through the various pathways. The gas density and temperature inside the sources and ISE are approximately spatially homogenous, since the dimensions of these structures are much larger than those of the gas escape pathways. Additionally, for volatile species we estimate the time-constants in these regions to be of the order of milliseconds (Teolis et al. 2010), and much less than the INMS integration period (IP) of $31 \mathrm{~ms}$. Therefore the gas flows and resulting densities are always in approximate steady state during the measurements. 
Considering first the CS, we write the steady-state expression equating ingoing and outgoing gas flows as follows:

$$
\text { Flow in }=\text { Flow out } \therefore \sum_{i} n_{i} v_{i} a_{i} t_{i}=n_{s} v_{s} \sum_{i} a_{i}^{\prime} t_{i}^{\prime},
$$

where $n_{s}$ is the molecule number density in the source and $n_{i}$ is the density outside the source at pathway $i$. Here we model every conduction pathway as consisting of (i) two apertures with areas $a_{i}$ and $a_{i}^{\prime}$ opposite and adjacent to the closed source and (ii) internal structure with transmission probabilities $t_{i}$ and $t_{i}^{\prime}$ for particles to pass through the pathway. $v_{i}$ and $v_{s}$ are the average magnitude of the molecule velocity components projected into the apertures of $i$ from outside and inside the source. We consider $v_{s}$ to be independent of $i$ due to the approximate spatial homogeneity of source density and temperature. We estimate transmittances of internal conduction pathways, i.e. the lens system and electron gun structures, with the standard analytical expressions for slits and cylinders (Dushman and Lafferty 1962), and that of the CS inlet exposed directly to the ram flux, with Monte Carlo calculations (Appendix E).

Starting from the flow balance expression Eq. (A.1) with $A=a t$ we solve for the source density:

$$
n_{s}=\frac{\sum_{i} n_{i} v_{i} A_{i}}{v_{s} \sum_{i} A_{i}^{\prime}},
$$

where $A$ is the 'effective' area; related to gas conductance $C$ by a factor of the average aperture projected speed, i.e., $C=v A$. We note that $v$ is (i) $1 / 4$ of the average molecule speed for internal components exposed to an isotropic velocity distribution, and (ii) a function of spacecraft speed and pointing and ambient gas temperature $T_{a}$ for components exposed to the ram flux. The denominator $\sum_{i} A_{i}^{\prime}$ is the total effective area to exit the close source, which contains the contribution of the CS inlet $\left(A_{0}\right)$, the lens system plus ion nozzle $\left(A_{2 a} \& A_{2 b}\right)$, and both electron gun structures $\left(2\left[A_{3 a} \&\left(2 A_{3 b}+A_{3 c}+4 A_{3 d}\right)\right]\right)$ :

$$
\sum_{i} A_{i}^{\prime}=A_{01}+A_{2 a} \& A_{2 b}+2\left[A_{3 a} \&\left(2 A_{3 b}+A_{3 c}+4 A_{3 d}\right)\right]
$$

where $\&$ denotes effective areas in series, i.e.,

$$
A_{X} \& A_{Y}=\left(A_{X}^{-1}+A_{Y}^{-1}\right)^{-1} \text {. }
$$

We include subscripts ' 0 ' and ' 1 ', e.g. $A_{0} \rightarrow A_{00}$ or $A_{01}$ where necessary to indicate ( 0 ) ram flux entering the conduction pathway, or (1) thermal gas escaping through the pathway to space, respectively. The Eq. (A.2) numerator is the sum of all ingoing fluxes, consisting of one contribution from the ambient environment $n_{a} v_{00} A_{00}$ (i.e. via the CS inlet) and another from the ISE $n_{i s e} v_{i s e}\left(A_{2 a} \& A_{2 b}+2\left[A_{3 a} \&\left(2 A_{3 b}+A_{3 c}+4 A_{3 d}\right)\right]\right)$, where $n_{\text {ise }}$ denotes the ISE density. We may therefore write Eq. (A.2) in the form

$$
n_{s}=n_{a}\left(\frac{v_{00}}{v_{s}}\right) R_{0}+n_{\text {ise }}\left(\frac{v_{\text {ise }}}{v_{s}}\right) R_{1} \text {, }
$$

where

$$
\begin{aligned}
& R_{0}=\frac{A_{00}}{A_{01}+A_{C S}}, \\
& R_{1}=\frac{A_{C S}}{A_{01}+A_{C S}},
\end{aligned}
$$


and the total effective area $A_{C S}=3.32 \mathrm{~mm}^{2}$ between the closed source and ISE is

$$
A_{C S}=A_{2 a} \& A_{2 b}+2\left[A_{3 a} \&\left(2 A_{3 b}+A_{3 c}+4 A_{3 d}\right)\right],
$$

with the effective areas on the RHS given in Table $1 . R_{1}$ is a constant number $\left(R_{1}=0.6254\right)$ due to its dependence solely on internal conductances, and is unaffected by spacecraft speed and pointing. Notably, $A_{C S}$ exceeds $A_{01}=1.989 \mathrm{~mm}^{2}$ (Table 1), and therefore neutral gas is more likely to "leak" from the CS into the rest of the instrument, than the escape back out through the CS inlet.

The ISE steady-state density also satisfies a flow balance expression of the same form as Eq. (A.2):

$$
n_{\text {ise }}=\frac{\sum_{i} n_{i} v_{i} A_{i}}{v_{i s e} \sum_{i} A_{i}^{\prime}},
$$

and like the expression for $n_{s}$ given in Eq. (A.5), similar considerations yield an analogous expression for $n_{\text {ise }}$ :

$$
n_{\text {ise }}=n_{a}\left(\frac{v_{00}}{v_{\text {ise }}}\right) R_{2}+n_{a}\left(\frac{v_{50}}{v_{\text {ise }}}\right) R_{3} \text {, }
$$

where

$$
\begin{aligned}
& R_{2}=\frac{A_{40}+A_{60} \& A_{7}+A_{00} \& A_{C S}}{A_{41}+A_{61} \& A_{7}+A_{51}+A_{01} \& A_{C S}} \\
& R_{3}=\frac{A_{50}}{A_{41}+A_{61} \& A_{7}+A_{51}+A_{01} \& A_{C S}} .
\end{aligned}
$$

Substituting Eq. (A.9) into (A.5) we obtain

$$
n_{s}=\left\{\left(\frac{v_{00}}{v_{s}}\right) R_{0}+\left[\left(\frac{v_{00}}{v_{s}}\right) R_{2}+\left(\frac{v_{50}}{v_{s}}\right) R_{3}\right] R_{1}\right\} n_{a} .
$$

Considering the ambient gas to have a Maxwell-Boltzmann velocity distribution with temperature $T_{a}$, the speed ratios $v_{00} / v_{s}$ and $v_{00} / v_{i s e}$ are

$$
\begin{aligned}
& \frac{v_{00}}{v_{s}}=\left(\frac{T_{a}}{T_{s}}\right)^{\frac{1}{2}} F, \\
& \frac{v_{00}}{v_{\text {ise }}}=\left(\frac{T_{a}}{T_{\text {ise }}}\right)^{\frac{1}{2}} F .
\end{aligned}
$$

Here $T_{s}$ and $T_{i s e}$ are the CS and ISE gas temperatures, and

$$
\begin{aligned}
F & =\frac{2}{\pi} \int_{-\infty}^{\infty} e^{-s_{x}^{2}} d s_{x} \int_{-\infty}^{\infty} e^{-s_{y}^{2}} d s_{y} \int_{-S}^{\infty}\left(s_{z}+S\right) e^{-s_{z}^{2}} d s_{z}=e^{-S^{2}}+\sqrt{\pi} S[1+\operatorname{erf}(S)], \\
S & =S_{0} \cos \theta
\end{aligned}
$$

where $s_{x}, s_{y}$ and $s_{z}$ are the molecule velocity components in units of $u_{0}=\sqrt{2 k T_{a} / m_{f}}$ (the most probable speed of the ambient gas molecules in the ambient frame) and the integral $F$ is evaluated over the velocity half space pointed toward the conduction pathway. Here 
$\theta$ is the ram angle (Fig. 3) with respect to the rest frame of the ambient gas, and $S_{0}$ is the spacecraft speed $u$ in units of $u_{0}$, i.e.,

$$
S_{0}=u \sqrt{m_{f} / 2 k T_{a}}=u / u_{0},
$$

where $m_{f}$ is the molecular mass of species $f$. Note that we do not assume an equivalence of $F$ with the CS ram enhancement factor, as was done in the original INMS calibration model (Appendix E).

We have distinguished the average projected velocity $v_{00}$ into the antechamber and open source from the projected velocity into the vent $v_{50}$, which points at 90 degrees to the closed and open sources (Figs. $1 \& 3$ ). An expression of the same form as Eq. (A.13) can be written for the speed ratios $v_{50} / v_{s}$ and $v_{50} / v_{\text {ise }}$, by replacing $\theta$ with the angle $\theta^{\prime}=\cos ^{-1}(\cos \phi \sin \theta)$ of the vent normal to the spacecraft direction of motion in the ambient frame (the vent ram angle):

$$
\begin{gathered}
\frac{v_{50}}{v_{s}}=\left(\frac{T_{a}}{T_{s}}\right)^{\frac{1}{2}} F^{\prime} \\
\frac{v_{50}}{v_{i s e}}=\left(\frac{T_{a}}{T_{i s e}}\right)^{\frac{1}{2}} F^{\prime} \\
F^{\prime}=e^{-S^{\prime 2}}+\sqrt{\pi} S^{\prime}\left[1+\operatorname{erf}\left(S^{\prime}\right)\right] \\
S^{\prime}=S_{0} \cos \theta^{\prime}=S_{0} \cos \phi \sin \theta
\end{gathered}
$$

where we define the azimuthal angle $\phi$ as the left-handed angle of the spacecraft velocity about $+X$ from $-Y$ in spacecraft coordinates (Fig. 3).

Substitution of Eqs. (A.12a) and (A.15a) into (A.11) yields

$$
n_{s}=\left(\frac{T_{a}}{T_{s}}\right)^{\frac{1}{2}} D_{s} n_{a},
$$

where

$$
D_{s}=\left(R_{0}+R_{2} R_{1}\right) F+R_{3} R_{1} F^{\prime}
$$

is the closed source ram enhancement factor (contrary to the original calibration where $D_{s}=$ $F$, see Appendix E). Likewise, substitution of Eqs. (A.12b) and (A.15b) into (A.9) gives

$$
n_{\text {ise }}=\left(\frac{T_{a}}{T_{i s e}}\right)^{\frac{1}{2}} D_{i s e} n_{a},
$$

where

$$
D_{\text {ise }}=R_{2} F+R_{3} F^{\prime} \text {. }
$$

The detector count rate $X$ [the number of counts divided by the $31 \mathrm{~ms}$ IP minus background, after correcting for instrumental effects, Magee et al. 2009] in CSN (closed source neutral) mode for species $f$ is

$$
X_{f}=\left(\frac{T_{s}}{T_{0}}\right)^{\frac{1}{2}} s_{f} n_{s f}
$$


where $s_{f}$ is the CSN sensitivity for $f$. The ratio $\sqrt{T_{s} / T_{0}}$ appears in Eq. (A.21) because laboratory $s_{f}$ calibrations are performed with respect to the equilibrium density at room temperature $T_{0}(\sim 293 \mathrm{~K})$ surrounding the instrument, which is different from $n_{s}$ by a factor $\sqrt{T_{s} / T_{0}}$. The combination of Eqs. (A.17) and (A.21) yields the final expressions for count rate and ambient density:

$$
X_{f}=s_{f}\left(\frac{T_{a}}{T_{0}}\right)^{\frac{1}{2}} D_{s f} n_{a f}
$$

or

$$
n_{a f}=\frac{1}{D_{s f}}\left(\frac{T_{0}}{T_{a}}\right)^{\frac{1}{2}} \frac{X_{f}}{s_{f}} .
$$

Similarly in open source neutral thermal (OSNT) mode, which measures the OS neutral density (Waite et al. 2004), we have

$$
X_{o s f}=\left(\frac{T_{o s}}{T_{0}}\right)^{\frac{1}{2}} s_{o s f} n_{o s f}
$$

with $X_{o s}, s_{o s}, T_{o s}$, and $n_{o s}$ the OSNT count rate, sensitivity, temperature and density, respectively. Writing an expression of the same form as Eq. (A.2) for the OS density, we have

$$
n_{o s}=\frac{\sum_{i} n_{i} v_{i} A_{i}}{v_{o s} \sum_{i} A_{i}^{\prime}}=\frac{v_{00} A_{60} n_{a}+v_{i s e}\left(A_{7}+A_{8}\right) n_{i s e}}{v_{o s}\left(A_{61}+A_{7}+A_{8}\right)},
$$

since the OS communicates with the ambient density through the OS collimator assembly (path 6 in Table 1, Fig. 4a), and with the ISE through (i) the OS assembly (path 7), and (ii) the OS collimator assembly (path 8). Substituting Eq. (A.9) for the ISE density into Eq. (A.24) above, we obtain

$$
n_{o s}=\frac{\left(\frac{v_{00}}{v_{o s}}\right) A_{60}+\left(A_{7}+A_{8}\right)\left(\frac{v_{00}}{v_{o s}}\right) R_{2}+\left(A_{7}+A_{8}\right)\left(\frac{v_{50}}{v_{o s}}\right) R_{3}}{A_{61}+A_{7}+A_{8}} n_{a} .
$$

Analogous to Eqs. (A.12a), (A.12b) and (A.15a), (A.15b) for the CS, we have for the OS

$$
\begin{aligned}
& \frac{v_{00}}{v_{o s}}=\left(\frac{T_{a}}{T_{o s}}\right)^{\frac{1}{2}} F \\
& \frac{v_{50}}{v_{o s}}=\left(\frac{T_{a}}{T_{o s}}\right)^{\frac{1}{2}} F^{\prime}
\end{aligned}
$$

Therefore Eq. (A.25) becomes

$$
n_{o s}=\left(\frac{T_{a}}{T_{o s}}\right) D_{o s} n_{a}
$$

where

$$
D_{o s}=R_{4} F+R_{5} F^{\prime}
$$


Fig. 16 Schematic showing the rotation of the vent away from ram during the T85 and T88

Titan encounters, together with a T85 plot showing the drop in signal (mass 28 OSNT counts per IP) 200-100 sec before closest approach as $\theta^{\prime}$ exceeds $90 \mathrm{deg}$. Axes indicate spacecraft coordinates: the CS inlet and OS collimator point out of the page (i.e., in $-X$ ). Spikes are caused by thruster firings. Negative spikes are due to scattering of the ambient $\mathrm{N}_{2}$ away from the INMS vent by the thruster exhaust when $\theta^{\prime}<90 \mathrm{deg}$, while positive spikes for $\theta^{\prime}>90 \mathrm{deg}$ are due to scattering of ambient $\mathrm{N}_{2}$ toward the INMS

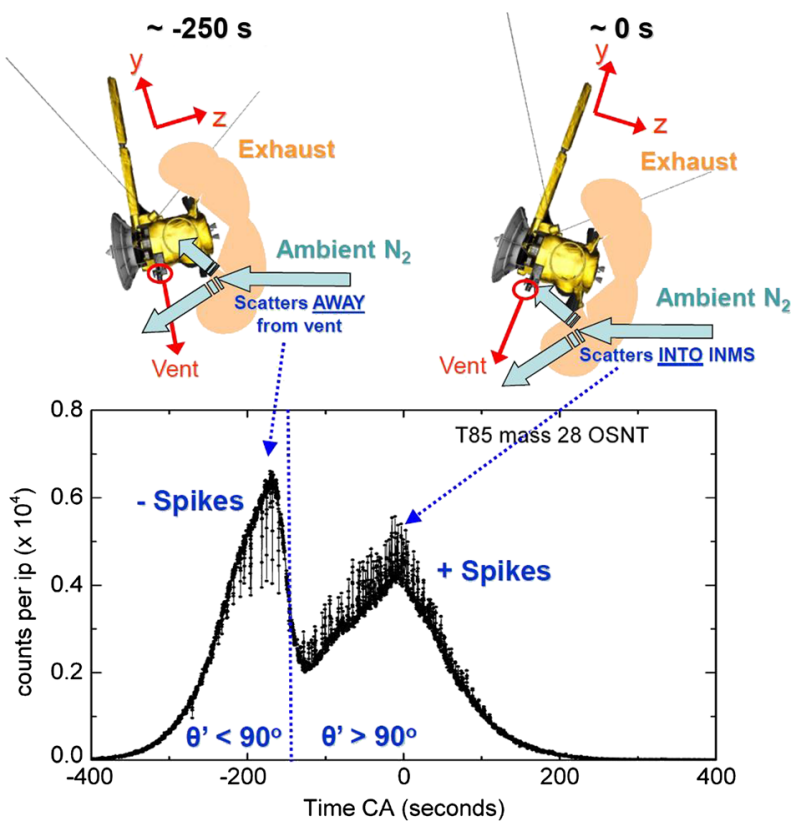

and

$$
\begin{aligned}
& R_{4}=\frac{A_{60}+\left(A_{7}+A_{8}\right) R_{2}}{A_{61}+A_{7}+A_{8}}, \\
& R_{5}=\frac{\left(A_{7}+A_{8}\right) R_{3}}{A_{61}+A_{7}+A_{8}} .
\end{aligned}
$$

From Eqs. (A.23) and (A.27), we obtain the OSNT count rate in terms of the ambient density:

$$
X_{o s f}=s_{o s f}\left(\frac{T_{a}}{T_{0}}\right)^{\frac{1}{2}} D_{o s} n_{a f} .
$$

Though the INMS rarely operates in OSNT mode, data acquired in this mode during the T85 and T88 Titan encounters are useful to the present analysis as we discuss below.

\section{Appendix B: In Flight Model Calibration: Path 7}

The OS assembly conductance (path 7) is cumbersome to model owing to the complexity of this section and limited detail of available drawings. Rather than estimate this pathway analytically or by Monte Carlo simulations, we used in flight data from the T85 and T88 flybys on 24 July and 29 Nov, 2012 during which the vent ram angle $\theta^{\prime}$ was swept from below to above $90 \mathrm{deg}$ (Fig. 16). This spacecraft pointing provided a unique opportunity to calibrate the model against its two limiting cases: i.e., entry of gas flux predominantly into (i) the CS inlet and OS collimator, or (ii) the vent. During both encounters INMS measured the CS and OS densities, using the CSN and OSNT modes, in only three channels to allow high $(0.1 \mathrm{sec})$ time resolution: 2,16 and $28 \mathrm{u}$ per charge; corresponding mostly to Titan 
Fig. 17 (a) OSNT counts at 2, 16 and 28 u per charge during the T85 flyby, corresponding mostly to Titanian $\mathrm{H}_{2}, \mathrm{CH}_{4}$ and $\mathrm{N}_{2}$, respectively. The ambient density for each species can be obtained from the count rate $X_{\text {osf }}$ (counts divided by the $0.031 \mathrm{sec}$ integration period) by Eq. (A.30) (and, for CSN data, by

Eq. (A.22a), (A.22b)). Exhaust from thruster firings is the source of the positive $\mathrm{H}_{2}$ spikes.

Negative $\mathrm{N}_{2}$ and $\mathrm{CH}_{4}$ spikes are due to scattering of the ambient atmosphere by the exhaust plume, as shown in Fig. 16 (by comparison $\mathrm{N}_{2}$ from the thrusters contributes negligibly to signal spikes). (b) Same as (a) for CSN signals
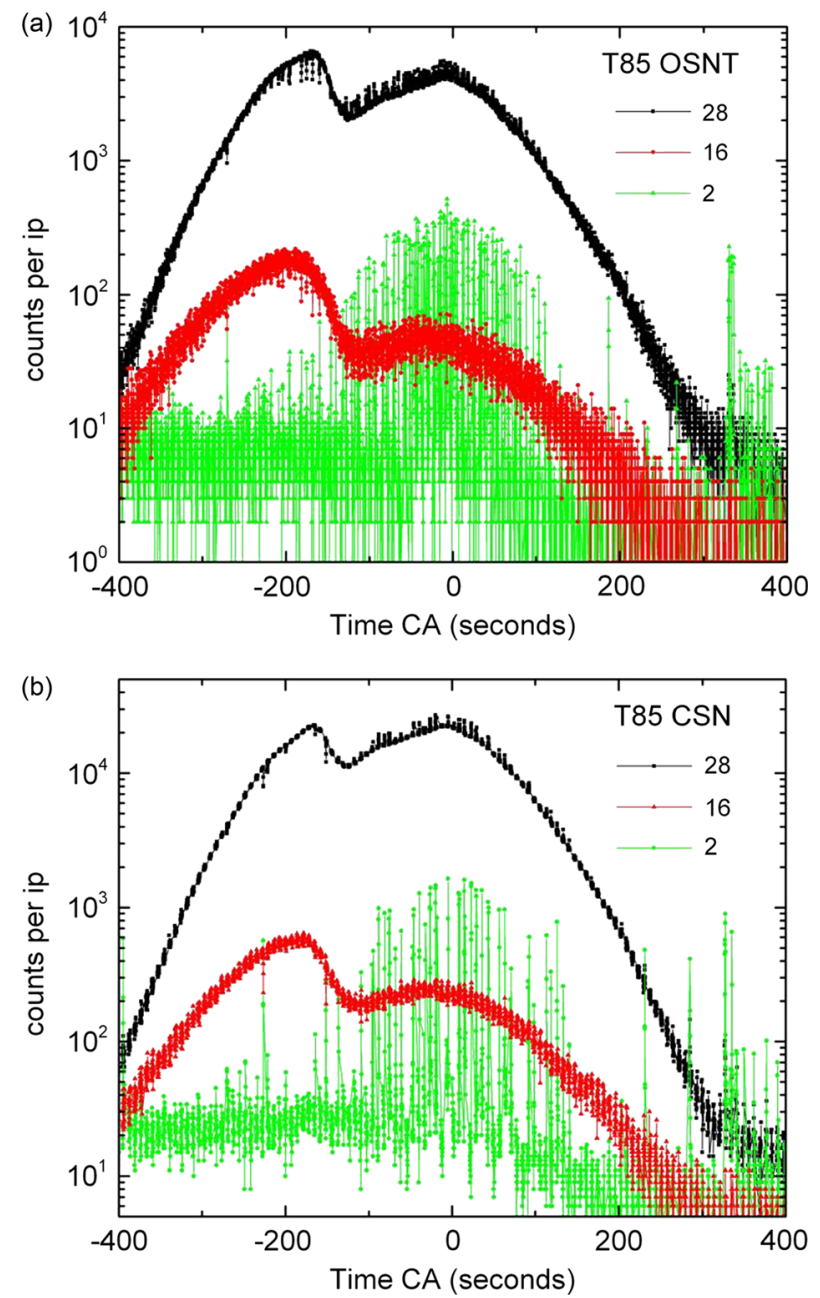

atmospheric $\mathrm{H}_{2}, \mathrm{CH}_{4}$ and $\mathrm{N}_{2}$ (Fig. 17). The Titan densities are also uncertain by a factor of (roughly) three due to atmospheric scale height differences between flybys, depending on time and positional variability of solar and/or plasma heating (Cui et al. 2009; Magee et al. 2009; Westlake et al. 2011). We therefore cancel the effect of Titan's densities by plotting the ratio of CSN and OSNT, obtained by dividing Eq. (A.30) by (A.22a):

$$
\frac{X_{o s f}}{X_{f}}=\frac{s_{o s f} D_{o s}}{s_{f} D_{s f}},
$$

where $s_{f}=4.05 \times 10^{-10} \mathrm{~m}^{3} / \mathrm{s}$ for ${ }^{14} \mathrm{~N}_{2}$. Additionally $X_{f}$ and $X_{o s f}$ are affected equally by spacecraft shadowing effects which are significant at T85 and T88 (Appendix D), and therefore this source of uncertainty is also canceled by the ratio. We have fit Eq. (B.1) to the ratio of OSNT to CSN counts at 28 u per charge for T85 and T88, with $A_{7}$ and the OSNT sensitivity factor $s_{o s f}$ as the fitting parameters. We fit $s_{o s f}$ since-by comparison to the $s_{f}$ factors-the OSNT sensitivity is much less well characterized, with only one measurement available for $\mathrm{N}_{2}$ (Waite et al. 2004). Our fit yields $s_{\text {osf }}=1.33( \pm 0.12) \times 10^{-10} \mathrm{~m}^{3} / \mathrm{s}$ for 
Fig. 18 Ratio of the OSNT to $\mathrm{CSN} \mathrm{N}_{2}$ signal in the mass 28 channel, during the T85 (circles) and T88 (squares) flybys, together with the optimal model (Eq. (B.1)) fit with OSNT sensitivity

$s_{\text {osf }}=1.33 \times 10^{-10} \mathrm{~m}^{3} / \mathrm{s}$ and OS-ISE effective area $\mathrm{A}_{7}=1.55 \times 10^{-7} \mathrm{~m}^{2}$ (solid lines). Dashed line: model prediction with $A_{7}$ three times optimal. Dotted line: same with $A_{7}=0$. The step like decrease in the ratio occurs, as anticipated by the model, when the vent ram angle exceeds 90 degrees. One can see that the success in fitting the data is not very sensitive to uncertainty in $A_{7}$

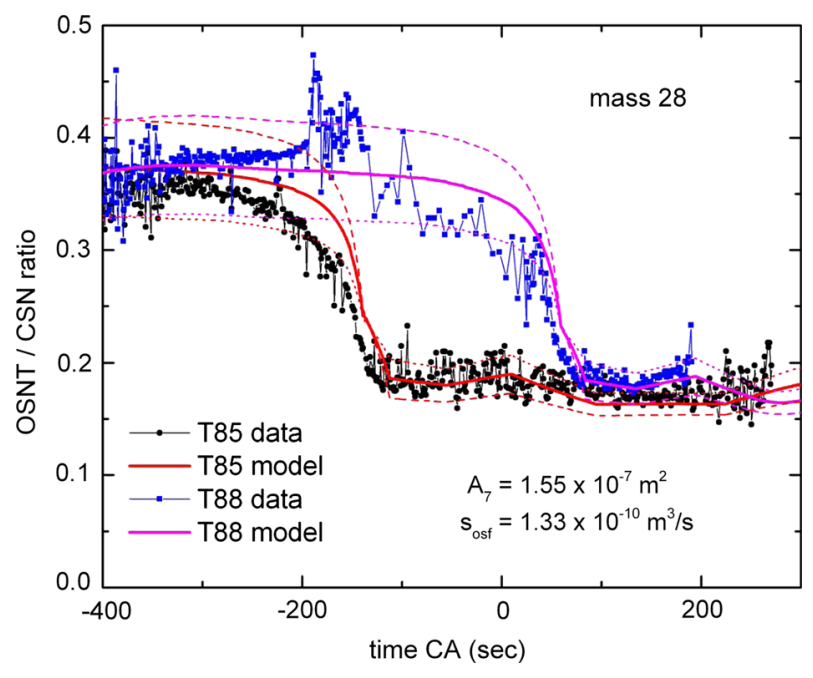

${ }^{14} \mathrm{~N}_{2}$, which agrees within error with the (Waite et al. 2004) value of $1.23 \times 10^{-10} \mathrm{~m}^{3} / \mathrm{s}$ (their Table IV). We note that the $s_{f}$ and $s_{\text {osf }}$ values given here have been reduced by the correction factor by $\beta=1.55$, as discussed in Sect. 4 . In Fig. 18 we compare the ratio of OSNT to CSN counts at $28 \mathrm{u}$ per charge at T85 to Eq. (B.1) for different values of $A_{7}$, including the best fit of $1.5( \pm 0.8) \times 10^{-7} \mathrm{~m}^{2}$. As expected both flybys exhibit a step like decrease in the ratio when the vent ram angle approaches $90 \mathrm{deg}$. Above $90 \mathrm{deg}$ the dominant gas source is through the CS inlet and OS collimator, rather than the vent, resulting in a different distribution of densities between the CS, OS and ISE. The fit is shifted up/down on Fig. 18 by $s_{o s f}$ since this factor is just a multiplicative constant in Eq. (B.1), while the magnitude of the step is controlled by $\mathrm{A}_{7}$. As shown in Fig. 18 the success of the fit is in fact rather insensitive to uncertainty in $\mathrm{A}_{7}$, since most gas flow between the ISE and ambient environment is through pathways 4 and 5 (bypassing path 7) due to their large effective areas (Table 1).

Two other flybys: T47 and T96, had similar spacecraft pointing (Fig. 5) to T85 and T88. T96 was at higher altitude (1400 km closest approach) than T47, 85 and 88 (1023, 1012 and $1014 \mathrm{~km} \mathrm{CA}$, respectively), and therefore the density was lower, and the data noisier (Fig. 19). Nevertheless, we found that the T96 OSNT/CSN ratio behaves as predicted by the model. A fit of the ratio to T47 data was not possible since OSNT data was not acquired during this flyby.

\section{Appendix C: Ram Factor Empirical Function Fits}

Ambient neutral density estimates by way of Eq. (A.22b) require knowledge of $D$ versus $\theta, \phi$ and $S_{0}$. Since the ram factor modeling depends in part on the results of Monte Carlo simulations, $D_{s}$ must be estimated from lookup tables. The INMS team plans in the near future to provide the lookup tables on the Planetary Data System. However we have also fit the $R_{0}, R_{2}$, and $R_{3}$ values from the tables with the empirical fitting functions given below, from which $D_{s}$ can be directly calculated (Eq. (A.18)). The empirical functions can serve as a convenient alternative to the tables. Except for $R_{1}$ which is constant, the functions are parameterized with respect to the components $S_{0}, \theta, \phi$ of the spacecraft velocity vector in the 


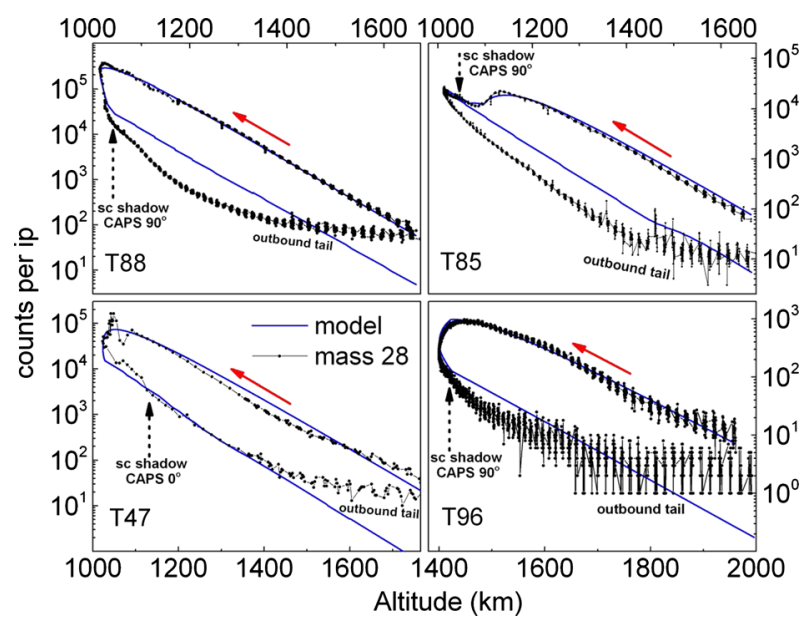

Fig. 19 T47, 85, 88 and 96 CSN mass 28 counts vs. altitude between -400 and 400 sec from CA, compared with a fit of the new model using (as an approximation of Titan's atmosphere) an exponential density vs. altitude $n(x)=\mathrm{C} \times \exp (-x / H)$ with $\log _{10}(\mathrm{C})=22.44$ (T47), 21.23 (T85), 20.99 (T88) and 20.35 (T96), and $H=336$ (T47), 385 (T85), 396 (T88) and 461 (T96) in km. Solid arrow: inbound branch. Dashed arrow: start of spacecraft shadowing by CAPS; the model over-estimates the signal after this point. However at high altitude the model under-estimates the signal again due to residual $\mathrm{N}_{2}$ in the instrument

gas rest frame. Therefore, aside from the CSN (or OSNT) count rate and species sensitivity, three inputs: $S_{0}, \theta$ and $\phi$, are necessary to obtain ambient neutral densities along Cassini's trajectory. First, for completeness we rewrite Eqs. (A.13), (A.16), (A.18) and (A.22b) for the ram factors and species density in terms of $R$ and $F$, below:

$$
\begin{aligned}
F & =e^{-S^{2}}+\sqrt{\pi} S[1+\operatorname{erf}(S)] \\
S & =S_{0} \cos \theta \\
F^{\prime} & =e^{-S^{\prime 2}}+\sqrt{\pi} S^{\prime}\left[1+\operatorname{erf}\left(S^{\prime}\right)\right] \\
S^{\prime} & =S_{0} \cos \phi \sin \theta \\
D_{s} & =\left(R_{0}+R_{2} R_{1}\right) F+R_{3} R_{1} F^{\prime} \\
n_{a f} & =\frac{1}{D_{s f}}\left(\frac{T_{0}}{T_{a}}\right)^{\frac{1}{2}} \frac{X_{f}}{S_{f}} .
\end{aligned}
$$

Here the constant value of $R_{1}$ is estimated from the ram factor modeling to be 0.6254 .

The empirical functions for $R_{0}, R_{2}$, and $R_{3}$, which feed into the above expressions, are as follows:

$$
\begin{aligned}
& R_{0}=Q_{1}+\left[Q_{2}-Q_{3} \exp \left\{-Q_{5}\left(S_{0}+Q_{4}\right)^{2}\right\}\right] \exp \left\{-q_{1}\left(Q_{6}\left(\theta-Q_{8}\right)^{2}+Q_{7}(\theta \tan \phi)^{2}\right)\right\} \\
& \quad \text { for } \pi / 2 \leq \phi \leq 3 \pi / 2 \\
& R_{0}=Q_{1} \quad \text { for } 0<\phi<\pi / 2 \text { or } 3 \pi / 2<\phi<2 \pi \\
& R_{2}=G_{1}+G_{2}\left[1-\exp \left(-G_{3} S_{0}\right)\right] \exp \left(-G_{4} \theta^{2}\right)+G_{5} \exp \left(-G_{3} S_{0}\right) \\
& R_{3}=H_{4} h_{1} h_{2} h_{4} h_{9} \quad \text { for } 0<\phi<\pi / 2 \text { or } 3 \pi / 2<\phi<2 \pi
\end{aligned}
$$


where the values of the fitting constants, written in uppercase, are listed in Table 2, and the lowercase quantities are given by

$$
\begin{aligned}
& q_{1}=Q_{9}-Q_{10} \exp \left(-Q_{11} S_{0}^{Q_{12}}\right) \\
& h_{7}=1-\exp \left(-H_{10} S_{0}\right) \\
& h_{8}=h_{7} \cos ^{4} \phi \\
& h_{9}=1+H_{12} h_{7}^{H_{11}} \cos ^{4} \phi \\
& h_{3}=1+H_{2}(\cos (4 \phi)-1) \\
& h_{5}=1-H_{3}\left(h_{7} \cos (4 \phi)-1\right) \\
& h_{1}=1+H_{5} h_{7} \sin \theta \cos \phi \\
& h_{2}=1+H_{6} h_{7} h_{8} \sin \left(4 h_{5}\left(\theta-H_{8}\right)-H_{1} h_{3}\right) \\
& h_{6}=1 /\left(H_{9}+H_{13} h_{7} \cos ^{2 h_{7}} \phi\right) \\
& h_{4}=1+H_{7}\left(h_{6}-1\right) .
\end{aligned}
$$

Note that we did not fit $R_{3}$ for $\pi / 2 \leq \phi \leq 3 \pi / 2$ since the behavior is complex (requiring lengthy fitting functions) while the actual contribution of $R_{3}$ to $D_{s}$ is negligible in this angular range. Though not strictly correct, it is convenient to set $R_{3}=0.55$ at these angles to ensure continuity of $R_{3}$ at $\phi=\pi / 2$ and $3 \pi / 2$. Finally, we have also fit $A_{60}$ :

$$
A_{60}=M_{1}\left[1-\exp \left(-M_{2} S_{0}^{2}\right)\right]\left(1+M_{3} \theta^{2}\right)^{-1}+M_{4} \exp \left(-M_{2} S_{0}^{2}\right)+M_{5}
$$

which (together with $R_{0}, R_{1}, R_{2}$ and $R_{3}$ given above) is required to calculate $R_{4}$ and $R_{5}$ by Eqs. (A.29a), (A.29b) and, in turn, $D_{o s}$ by Eq. (A.28).

\section{Appendix D: Spacecraft Shadowing}

One can forward model the expected $\mathrm{N}_{2}$ count rate by entering a reasonable density vs. altitude function into Eqs. (A.22a) and (A.30). We use here $n(x)=\mathrm{C} \times \exp (-x / H)$ with fitting parameters $C$ and $H$, where the scale height $H$ is approximated as constant [as adequate for our purposes, Westlake et al. 2011]. However using the T85, 88 and 96 inbound densities to constrain the fit of $X_{s}$ and $X_{o s}$, we found that the model over-estimates the signal after $\theta^{\prime}$ exceeds $\sim 90 \mathrm{deg}$ by a factor of $\sim 6$ in the worst case (Fig. 19). For T47 the deviation from the model is comparatively minor (Fig. 19), and only after $\theta^{\prime}$ exceeds $\sim 110 \mathrm{deg}$. The explanation is the actuator angle of the rotatable Cassini Plasma Spectrometer (CAPS), which lies beneath INMS on the spacecraft deck. Contrary to T47 when CAPS was actuated to a rotation angle of $0 \mathrm{deg}$ for most of the encounter $(+/-10 \mathrm{~min})$, the rotation angle was near 90 deg (Fig. 20) during the T85, T88 and T96 flybys, which was the final position when CAPS went offline on June 2, 2012 prior to T85. At 90 deg rotation angle the CAPS instrument protrudes farther into the INMS ram line of sight shortly after $\theta^{\prime}$ passes $90 \mathrm{deg}$ (Fig. 20), i.e., approximately the same time as the $\mathrm{N}_{2}$ signal drops significantly below the expected level.

In Fig. 5 we show the $\theta, \varphi$ ram directions shadowed by the spacecraft, overlaid onto an estimate of the CSN ram factor $D_{s}$ for $S_{0}=20$ (typical of a Titan flyby). One can see for 
Fig. 20 Top: Diagram of INMS (yellow) on the spacecraft deck (green), showing the view obstruction by the CAPS instrument (red) during the T85 Titan encounter, with the time evolution of the ram direction between $-400 \mathrm{sec}$ and $400 \mathrm{sec}$ (from Titan closest approach) indicated by the lines in $5 \mathrm{sec}$ intervals. CAPS was rotated to $\sim 90$ degrees actuator angle as shown, and the lines are colored white when CAPS blocks the view of INMS in the ram direction. Bottom: Assuming for purpose of comparison a $0 \mathrm{deg}$ CAPS angle (in place of the correct T85 angle of $\sim 90 \mathrm{deg}$ )

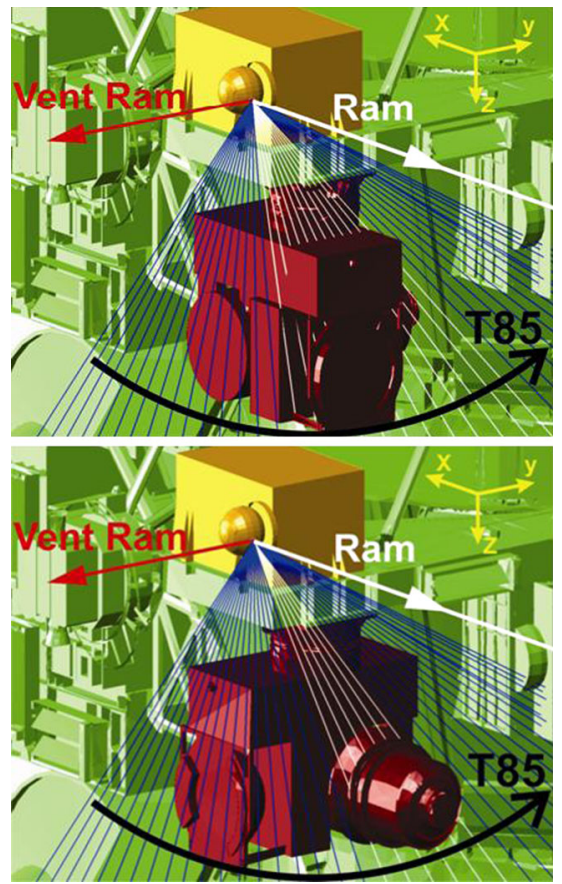

the most part the spacecraft shadow zone lies (by design) in the areas of low INMS sensitivity, i.e., far off ram. We do not currently include the effect of spacecraft shadowing in our ram factor modelling, i.e., in the estimates of the $D$ factors. This would require multiplication of $F$ and $F^{\prime}$ by spacecraft correction factors, obtained from modeling of (1) direct shadowing of the ram flux, and (2) secondary scattering from other spacecraft surfaces into the INMS. Direct shadowing becomes important for pointing within a few degrees of the spacecraft shadow zone, due to the small thermal spread of the incoming ambient molecule velocity vectors about the ram direction (assuming $S_{0} \gg 1$ ). Inside the shadow zone (i.e., ram from behind the spacecraft), secondary scattering from the spacecraft becomes the dominant contribution to the signal. Inclusion of the spacecraft correction factors is of course a possibility for future work, which would extend the range of validity of the ram factor modelling to all $4 \pi$ steradians of $\theta$ and $\varphi$. However, with the caveat that $S_{0}$ should be significantly greater than unity (essentially always true), we recommend for now that the ram factor modelling be used for angles for which no more than $5 \%$ of the ram flux is shadowed by the spacecraft. These angles are shown in Fig. 6. For a typical Titan flyby $S_{0}$ of 20 this requirement translates to a minimum angle of $\sim 3$ degrees of the pointing from the edge of spacecraft.

\section{Appendix E: Comparison to Original 'Ideal' Calibration Model}

The 'ideal' closed source that was the basis of the original calibration model was approximated as completely sealed, i.e., with the exception of the CS inlet as the only gas conduction pathway. With this assumption the analysis of Appendix A yields

$$
n_{s}=\frac{A_{00}}{A_{01}}\left(\frac{T_{a}}{T_{s}}\right)^{\frac{1}{2}} F n_{a},
$$


which is independent of the flux into the vent or OS collimator. The approximation $A_{00} \approx$ $A_{01}$ is made to the CS inlet since the combined shape is conductance limited mainly by the tube, which receives an isotropic molecular velocity distribution at both ends. This approximation breaks down for ram directions near the special angle [at $\theta^{\prime}=13^{\circ}, \varphi=180^{\circ}$, see Teolis et al. 2010] where the ram molecular beam is aimed from the entrance aperture directly into the tube (Fig. 5), i.e., without antechamber wall collisions to pre-isotropize the molecule velocities. However the original calibration model did not consider this case. Therefore Eq. (E.1) becomes

$$
n_{s} \approx\left(\frac{T_{a}}{T_{s}}\right)^{\frac{1}{2}} F n_{a},
$$

with the CSN detector count rate $X$ for species $f$ (Eq. (A.21)) given by

$$
X_{f}=\left(\frac{T_{s}}{T_{0}}\right)^{\frac{1}{2}} s_{f} n_{s f} .
$$

Hence

$$
X_{f} \approx s_{f}\left(\frac{T_{a}}{T_{0}}\right)^{\frac{1}{2}} F_{f} n_{a f},
$$

or

$$
n_{a f} \approx \frac{1}{F_{f}}\left(\frac{T_{0}}{T_{a}}\right)^{\frac{1}{2}} \frac{X_{f}}{s_{f}},
$$

and the correction factor to the ambient neutral densities from the original calibration is $F / D_{s}$ times the reduction factor $\beta=1.55 \pm 21 \%$ (Sect. 4):

$$
\text { correction factor }=\beta \frac{F}{D_{s}}=\beta \frac{F}{\left(R_{0}+R_{2} R_{1}\right) F+R_{3} R_{1} F^{\prime}},
$$

with $D_{s}$ from Eq. (A.18). One can see by comparing Eqs. (E.2) and (A.17) that the ram factor $D_{s}$ is equivalent to $F$ in the original calibration, unlike the revised ram factor model presented in this paper. The angular $\theta$ and $\phi$ dependence of the correction at $S_{0}=20$ is shown in Fig. 10. The factor exceeds unity because the new INMS calibration model is less sensitive than the original: the result of (i) the $s_{f}$ sensitivity reduction, and (ii) the gas leakage out of the CS now accounted for.

Analyzing 53 Titan flybys, we find that the closest approach densities are on average a factor of 2.2 greater with the new INMS calibration than with the original calibration. This value is equal (within error) to the limiting $S_{0} \gg 1$ (supra-thermal spacecraft speed) value of the $\theta=0$ (on ram) correction factor: also $2.2 \pm 23 \%$. This constant is useful (in CSN mode) for re-interpreting densities evaluated with the original calibration for flybys fixed near 0 degrees ram. Unlike the neutral densities obtained in CSN and OSNT modes (subject to the ram factor modeling discussed above), we emphasize that the INMS ion and neutral beam measurements (OSI and OSNB modes) do not involve wall collisions and thermalization of the molecules in the instrument, and are therefore only affected by the (angle independent) $s_{f}$ sensitivity correction constant discussed in Sect. 4. Many recent INMS publications have adopted a constant correction factor of 3 for the CSN neutral densities, in anticipation of the more detailed assessment given in this paper. This factor is now superseded by the more accurate value of $2.2 \pm 23 \%$ (which includes ram factor and $s_{f}$ sensitivity corrections) 
for on-ram flybys, or, as appropriate, the angle dependent correction. Likewise, a constant factor $1.55 \pm 21 \%$ (i.e., $s_{f}$ sensitivity correction only, no angle dependence) should now be adopted for OSI and OSNB. In the $\mathrm{S}_{0} \gg 1$ limit considered here the correction factors are independent of the gas species since all gases enter the INMS with equivalent (near perfect) collimation. The factors are applicable in the 'collisionless' regime below $\sim 4 \times 10^{-9} \mathrm{~kg} / \mathrm{m}^{3}$ $\left(\sim 8 \times 10^{16}\right.$ molecules $\left./ \mathrm{m}^{3}\right)$ in which ambient mean free paths are larger than the spacecraft in all directions (including the direction of motion); a condition satisfied at all Titan altitudes traversed by Cassini.

\section{Appendix F: Monte Carlo Modeling}

Here we give a more detailed description of the Monte Carlo modeling of the transmission factors used to calculate effective areas in the INMS ram factor modeling. We estimate the CS and vent transmittances $t_{0}$ and $t_{5}$, as well as transmittances $t_{4}$ between the ambient environment and ISE through OS collimator slits, $t_{6}$ between ambient and the OS, and $t_{8}$ between the OS and ISE through the OS-aperture and OS collimator slits, with separate Monte Carlo simulations (Table 1). All pathways except 8 are exposed to the ram flux, and therefore we estimate the angle/speed dependent ingoing and outgoing transmittances (i.e., $t_{i}$ and $t_{i}^{\prime}$, respectively) with separate simulations. Only one simulation was required for pathway 8 since the effective areas are equal in both directions, as required for all internal pathways in thermodynamic equilibrium: hence $A_{8}^{\prime}=A_{8}$, i.e., $t_{8}^{\prime}=\left(a_{8} / a_{8}^{\prime}\right) t_{8}$ (the same equality holds for internal pathways 2, 3 and 7).

To estimate the CS inlet and OS collimator inward (unprimed) transmission we initialized particles from randomly distributed points on their entrance aperture planes, with an isotropic Maxwell-Boltzmann velocity distribution. We shifted the distribution in velocity space by the spacecraft velocity vector, and scaled it by velocity, i.e., $f(v) \rightarrow v f(v)$ such that particles are initialized according to the arrival rate at the aperture (Greenwood 2002). By contrast we subdivided the vent aperture into 20 flat sub-planes to approximate its curved surface, evaluating the number of molecules and the velocity vector distributions for each individual plane according to the portion $v f(v)$ within the velocity space hemisphere seen by that plane. The ingoing directional distribution is completely characterized by $S_{0}, \theta$ and $\phi$, and therefore $t_{i}=t_{i}\left(S_{0}, \theta, \phi\right)$, where $i=0,4,5$ or 6 (Table 1). We allowed molecules to bounce randomly inside the structure in question, and molecules striking the walls were re-emitted according to a cosine angular distribution with respect to the surface normal (Steckelmacher 1986; Wenaas 1971). We did not consider intermolecular collisions, since the size scales are much smaller than the mean free paths at typical densities encountered by INMS. We estimated the transmission probability as the ratio of the number of particles transmitted through the exit areas (i.e. slits or apertures or both), to the number created at the initialization area. The statistical error is the inverse square root of the total number of transmitted particles.

Likewise we estimate the outgoing transmissions by initializing molecules at random positions on the exit area(s) with a cosine directional distribution, as appropriate for an isotropic flux transiting a plane (Greenwood 2002). Unlike $t_{i}$, the $t_{i}^{\prime}$ are constant numbers, with no dependence on $S_{0}, \theta$ or $\phi$, since we are now considering only the escape of the already thermally equilibrated gas. We follow the same procedure as for $t_{i}$, estimating $t_{i}^{\prime}$ as the ratio of molecules transmitted through the entrance aperture back into space, to the number entering the structure from the exit area.

In the CS inlet simulation we consider the antechamber and the tube together, as shown in Teolis et al. (2010). We simulate the OS collimator as a short tube representing the entrance 
aperture, in series with a tube representing the collimator assembly. The exit areas consist of the four axial collimator slits, the circular inner and outer ring slits, and the exit aperture (see Fig. 3 detail on OS collimator). We consider the slit depth by assigning a transmission factor to each slit, given by the Clausing factor for the slit of the given width and depth (Dushman and Lafferty 1962). Particles that fail to transmit through the slit are re-directed isotropically back into the collimator assembly from the location where they struck the slit (we ignore 'lateral drift' of the particles in the slit, since for a narrow slit the effect is small). Particles re-entering the collimator assembly from the ISE must also pass through the slits, with the possibility of being rejected back into the ISE.

An equally valid alternative method of estimating transmission factors is a surface mesh approach, i.e. to define a shape's two-dimensional transfer matrix $M_{k l}$ describing the vapor transfer rate between the mesh elements which depends on element area, inter-element distance and relative orientation. The instantaneous molecule surface coverage $\sigma_{k}$ is modified iteratively with a time step $\Delta t: \sigma_{k} \rightarrow \sigma_{k}+\Delta t \sum_{l} M_{k l} \sigma_{l}$. The transmission factor is estimated from the ratio of the outgoing to ingoing flux after the number of iterations is sufficient to

reach steady state, i.e., $\vec{\sigma}_{\text {steady }}=\lim _{N \rightarrow \infty}(\overleftrightarrow{I}+\Delta t \overleftrightarrow{M})^{N} \vec{\sigma}$. A detailed explanation is given by Teolis et al. (2010), who applied the surface mesh approach to simulate the propagation of the 'sticky' molecule $\mathrm{H}_{2} \mathrm{O}$ through the $\mathrm{CS}$ inlet. However the model is equally applicable to 'non-sticky' volatiles, and for example our calculations of CS inlet transmission $t_{0}$ with the surface mesh approach are in excellent agreement with the Monte Carlo simulations.

\section{Appendix G: Thruster Spikes and Inter-molecular Collisions}

Negative $\mathrm{N}_{2}$ spikes during the T85 and T88 encounters (Figs. 16 \& 17 for T85) were measured simultaneously with the firing times of Cassini's reaction control thrusters, which were used (instead of Cassini's three reaction wheels) to stabilize the spacecraft orientation against atmospheric drag. Each thruster had a different effect on the signal, with the most intense negative spikes produced by the $\mathrm{Y} 3$ and $\mathrm{Z} 3$ thrusters. As shown in Figs. 16 \& 17 for T85, the negative spikes gained intensity until $\theta^{\prime}$ exceeded $90 \mathrm{deg}$ (at $\sim-140$ and $+60 \mathrm{sec}$ for T85 \& 88, respectively), whereupon the spikes abruptly became positive. We performed collisional Monte Carlo simulations of the exhaust plume to investigate the cause of the negative spikes, taking into account the plume composition (assuming as an approximation complete stoichiometric conversion of the $\mathrm{N}_{2} \mathrm{H}_{4}$ propellant to $\left.\sim 2: 1 \mathrm{H}_{2}: \mathrm{N}_{2}\right)$, mass $(\sim 0.5 \mathrm{~g} / \mathrm{s}$ expelled from the thrusters), and thrust [ $\sim 0.69 \mathrm{~N}$ Rizvi 2014], and intermolecular collisions with the ambient atmosphere streaming past the spacecraft. We simulated the ambient atmosphere as a drifted $150 \mathrm{~K}$ Maxwellian distribution of $\mathrm{N}_{2}$ molecules, the spacecraft body as cylindrical with the conical high-gain antenna (HGA) at one end, and set the thruster positions and exhaust plume directions accordingly with respect to the HGA and INMS position on the spacecraft body. Thermal accommodation coefficients near unity are routinely observed by Earth-orbiting satellites (Bowman and Moe 2005), and we therefore assumed as an approximation full thermal accommodation of the impacting molecules, and scattered them from the spacecraft according to a 'diffuse' cosine ejection angle distribution. Additionally, we monitored the arrival angles of molecules at the INMS instrument position, and convolved onto the angle-dependent modelling described in the preceding sections to predict the change of CSN and OSNT count rates due to thruster firings.

The simulations demonstrated that the exhaust plume $\mathrm{N}_{2}$ density (e.g. $\sim 7 \times 10^{17} \mathrm{~N}_{2} / \mathrm{m}^{3}$, or $\sim 3 \times 10^{-8} \mathrm{~kg} / \mathrm{m}^{3}$, two meters from the thruster) is sufficient for scattering of a major 
Fig. 21 Visual snapshot of the Monte Carlo thruster plume simulation, showing thruster Z3 exhaust $\mathrm{N}_{2}$ (yellow) and scattered ambient (white) molecules in relation to the Cassini spacecraft, and INMS position and pointing during the T85 Titan encounter

Fig. 22 Fractional change in ambient $\mathrm{N}_{2}$ signal during T85 z3 thruster firings from the CSN (squares) and OSNT (circles) data, compared to the thruster exhaust simulation prediction. Here the simulation assumes a $3000 \mathrm{~K}$ thruster exhaust temperature with a $0.5 \mathrm{~g} / \mathrm{s}$ burn rate and $1500 \mathrm{~m} / \mathrm{s}$ ejection speed (yielding $0.69 \mathrm{~N}$ of thrust)
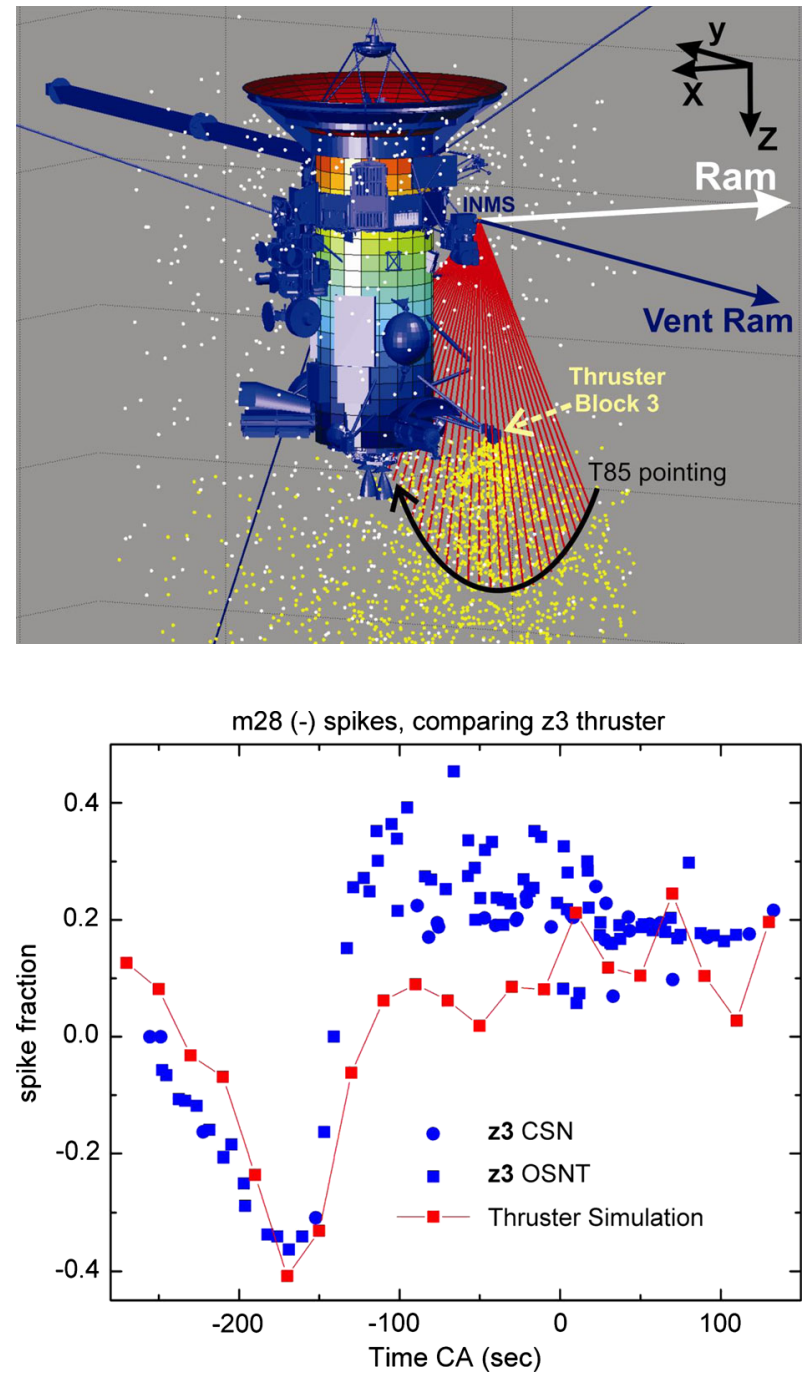

fraction of the incoming molecules away from the INMS by thrusters Y3 and Z3. The explanation is the near alignment of INMS's location along the $Z$ axis with thruster block 3 , which fires directly into the ram flux line of sight of the INMS vent (Figs. $16 \& 21$ ). However all three inlet pathways (CS, OS, and vent) are pointed obliquely to the ram direction for oblique $\theta^{\prime}$, and hence the instrument sees only a minor fraction of the ram flux after $\theta^{\prime}$ exceeds $90 \mathrm{deg}$. Therefore the thruster spikes become positive after $\theta^{\prime}$ passes $90 \mathrm{deg}$ since, as shown in Fig. 16, obstruction of the (small) direct flux by the exhaust plume is less significant than scattering of ambient $\mathrm{N}_{2}$ toward the inlet pathways.

The effectiveness of scattering (as much as $40 \%$ by the Y3 \& Z3 thrusters, Fig. 22) raises the question of whether the drag and/or torque on the spacecraft is significantly reduced by intermolecular collisions during thruster firing events. However our simulations indicate that the 'shadow' cast by the thruster plume (Fig. 23) covers only a small fraction of the spacecraft in the worst case, and often the ram direction does not aim the scattering shadow 


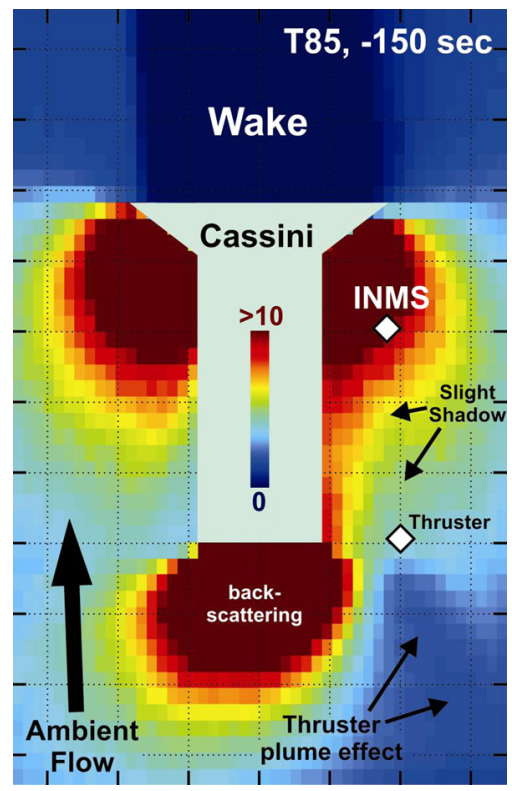

Fig. 23 Estimate of the ram enhancement factor (cross-section shown) vs. position (grid lines: 1 meter) from the thruster exhaust simulation, with the thruster plume parameters of Fig. 22, for the T85 ram direction (from below) and estimated ambient density $\left(\sim 6.7 \times 10^{14} \mathrm{~N}_{2} / \mathrm{m}^{3}\right)$ at $-150 \mathrm{sec}$ from closest approach to Titan, i.e., around the time when INMS observed negative $\mathrm{N}_{2}$ spikes. Cassini is simulated as a cylinder plus a cone for the high gain antenna. The ram factor shown here includes incident and scattered ambient $\mathrm{N}_{2}$, but excludes thruster exhaust. Backscattered $\mathrm{N}_{2}$ molecules produce drastic density ram enhancements near the spacecraft, except for the density wake downstream of the high gain antenna. Diamonds: thruster and INMS positions. The reduction in density near the thruster occurs as ambient molecules are scattered away by the exhaust plume. A slight "shadow" is also cast downstream of the thruster plume toward the INMS

at the spacecraft at all. Moreover, the shadowing tends to be largely mitigated since every scattering event produces a recoil $\mathrm{N}_{2}$ which has a good chance of striking the spacecraft. Clear evidence for recoils in the exhaust plume is seen in the positive $\mathrm{H}_{2}$ spikes (Fig. 17), which increase from $<10$ counts per IP (below the noise) to several hundred near $0 \mathrm{sec}$, as the higher atmospheric densities at closest approach scatter more thruster $\mathrm{H}_{2}$ back toward the INMS. During thruster firings we find that the thruster collisional correction to drag and torque is at most a few percent in the worst case, and therefore not a serious problem for AACS-based density estimates, nor a significant contributor to the difference with INMS.

A related consideration is the effect of inter-molecular collisions generally, i.e., without thruster plumes, since the mean free path as viewed in the spacecraft reference frame shortens to only $\sim 10$ meters at Titan closest approach densities $\left(\sim 10^{16}\right.$ molecules $/ \mathrm{m}^{3}$, or $\sim 5 \times 10^{-10} \mathrm{~kg} / \mathrm{m}^{3}$, at $1000 \mathrm{~km}$ altitude) for molecules forward-directed into the incoming ram flow. For example ambient $\mathrm{N}_{2}$ forward scattered off the spacecraft can re-collide in the spacecraft vicinity at closest approach densities, thereby slowing or scattering away a fraction of the incoming $\mathrm{N}_{2}$. However our simulations showed that the effect is small even at closest approach, and largely canceled by recoiling $\mathrm{N}_{2}$ re-striking the spacecraft. The simulations show a drop in drag coefficient at high density through conversion of the ram dynamic pressure into heat as the incoming gas slows in front of the spacecraft, but the effect is small, e.g., with only a $\sim 3 \%$ drop predicted at $2 \times 10^{-9} \mathrm{~kg} / \mathrm{m}^{3}\left(\sim 4 \times 10^{16}\right.$ molecules $\left./ \mathrm{m}^{3}\right)$ : approximately the maximum Titan density encountered in the mission (Figs. 7-9), imply- 
ing that the gas flow around the spacecraft is essentially collisionless. Moreover, significant collisional flow stagnation in front of the spacecraft would change the derived INMS and AACS densities in different proportions if intermolecular collisions were not accounted for, since the gas flux and drag force seen, respectively, by INMS and AACS, differ by a factor of (gas) velocity. Accordingly, the proportionality of the INMS and AACS densities over the entire range of Titan flyby densities (Fig. 7) indicates a constant drag coefficient within error at the relevant densities. We therefore conclude as expected that inter-molecular collisions cannot be a significant source of error between AACS and INMS.

\section{References}

J.M. Bell, S.W. Bougher, J.H. Waite, A.J. Ridley, B.A. Magee, K.E. Mandt, J. Westlake, A.D. DeJong, A. Bar-Nun, R. Jacovi, G. Toth, V. De La Haye, Simulating the one-dimensional structure of Titan's upper atmosphere: 1. Formulation of the Titan Global Ionosphere-Thermosphere Model and benchmark simulations. J. Geophys. Res. 115, E12002 (2010a)

J.M. Bell, S.W. Bougher, J.H. Waite, A.J. Ridley, B.A. Magee, K.E. Mandt, J. Westlake, A.D. DeJong, V. De La Haye, A. Bar-Nun, R. Jacovi, G. Toth, D. Gell, G. Fletcher, Simulating the one-dimensional structure of Titan's upper atmosphere: 2. Alternative scenarios for methane escape. J. Geophys. Res. 115, E12018 (2010b)

J.M. Bell, J.H. Waite, J.H. Westlake, S.W. Bougher, A.J. Ridley, R. Perryman, K. Mandt, Developing a self-consistent description of Titan's upper atmosphere without hydrodynamic escape. J. Geophys. Res. Space Phys. 119, 4957 (2014)

B.R. Bowman, K. Moe, Drag coefficient variability at $175-500 \mathrm{~km}$ from the orbit decay analyses of spheres, in Astrodynamics Specialist Conference. AIAA/AAS, Lake Tahoe, California, AAS 05-257 (2005)

F.J. Crary, B.A. Magee, K. Mandt, J.H. Waite, J. Westlake, D.T. Young, Heavy ions, temperatures and winds in Titan's ionosphere: Combined Cassini CAPS and INMS observations. Planet. Space Sci. 57, 1847 (2009)

T.E. Cravens, I.P. Robertson, J.H. Waite, R.V. Yelle, V. Vuitton, A.J. Coates, J.-E. Wahlund, K. Agren, M. Richard, V. de La Haye, A. Wellbrock, F.M. Neubauer, Model-data comparisons for Titan's nightside ionosphere. Icarus 199, 174 (2009)

J. Cui, R.V. Yelle, V. Vuitton, J.H. Waite, W.T. Kasprzak, D.A. Gell, H.B. Niemann, I.C.F. Muller-Wordag, N. Borggren, G.G. Fletcher, E.L. Patrick, E. Raaen, B.A. Magee, Analysis of Titan's neutral atmosphere from Cassini Ion Neutral Mass Spectrometer measurements. Icarus 200, 581 (2009)

S. Dushman, J.M. Lafferty, Scientific Foundations of Vacuum Technique (Wiley, New York, 1962)

R.C. Espiritus, R.H. Tolson, Determining Venusian Upper atmosphere Characteristics Using Magellan Attitude Control Data (Univelt, Inc., Albuquerque, 1995)

A.W. Feldman, J.A. Brown, E. Wang, S. Peer, A.Y. Lee, Reconstruction of Titan atmosphere density using Cassini attitude control flight data, in 17th AAS/AIAA Space Flight Mechanics Conference, Sedona, Arizona, USA, AAS 07-187 (2007)

M. Fulchignoni, Results on Titan's atmosphere structure by the Huygens atmospheric structure instrument, Space Week, Moscow (2007)

M. Fulchignoni, F. Ferri, F. Angrilli, A.J. Ball, A. Bar-Nun, M.A. Barucci, C. Bettanini, G. Bianchini, W. Borucki, G. Colombatti, M. Coradini, A. Coustenis, S. Debei, P. Falkner, G. Fanti, E. Flamini, V. Gaborit, R. Grard, M. Hamelin, A.M. Harri, B. Hathi, I. Jernej, M.R. Leese, A. Lehto, P.F.L. Stoppato, J.J. Lopez-Moreno, T. Makinen, J.A.M. McDonnell, C.P. McKay, G. Molina-Cuberos, F.M. Neubauer, V. Pirronello, R. Rodrigo, B. Saggin, K. Schwingenschuh, A. Seiff, F. Simoes, H. Svedhem, T. Tokano, M.C. Towner, R. Trautner, P. Withers, J.C. Zarnecki, In situ measurements of the physical characteristics of titan environment. Nature 438, 785 (2005)

M. Galand, R. Yelle, J. Cui, J.E. Wahlund, V. Vuitton, A. Wellbrock, A. Coates, Ionization sources in Titan's deep atmosphere. J. Geophys. Res. 115, A07312 (2010)

D.A. Gell, J.H. Waite, Characterization of the Cassini Ion Neutral Mass Spectrometer. Southwest Research Institute, report number 16063.02.120-1 (2015)

J. Greenwood, The correct and incorrect generation of a cosine distribution of scattered particles for MonteCarlo modelling of vacuum systems. Vacuum 67, 217 (2002)

A.Y. Lee, Model-based thruster leakage monitor for the Cassini spacecraft. J. Spacecr. Rockets 36, 745 (1999)

A.Y. Lee, G. Hanover, Cassini spacecraft attitude control system flight performance, in AIAA Guidance, Navigation, and Control Conference. American Institute of Aeronautics and Astronautics, San Francisco, California, USA, AIAA-2005-6269 (2005) 
A.Y. Lee, R. Lim, Evidence of temporal variation of Titan atmospheric density in 2005-2013, in at AIAA Guidance, AIAA Guidance, Navigation, and Control Conference (American Institute of Aeronautics and Astronautics, Boston, 2013)

A.Y. Lee, J. Wertz, In-flight calibration of the Cassini spacecraft inertia matrix. J. Spacecr. Rockets 39, 153 (2002)

D.S. Liechty, Cassini-Huygens aerodynamics with comparison to flight, in 25th International Symposium on Rarefied Gas Dynamics, Saint Petersburg, Russia (2006)

B.A. Magee, J.H. Waite, K.E. Mandt, J. Westlake, J. Bell, D.A. Gell, INMS derived composition of Titan's upper atmosphere: analysis methods. Planet. Space Sci. 57, 1895 (2009)

P.M. Mahhafy, K. Lai, An electrostatic quadrupole deflector for mass spectrometer applications. J. Vac. Sci. A 8, 3244 (1990)

K.E. Mandt, D.A. Gell, M. Perry, J.H. Waite, F.A. Crary, D. Young, B.A. Magee, J.H. Westlake, T. Cravens, W. Kasprzak, G. Miller, J.-E. Wahlund, K. Agren, N.J.T. Edberg, A.N. Heays, L.R. Brenton, S.T. Gibson, V. de la Haye, M.-C. Liang, Ion densities and composition of Titan's upper atmosphere derived from the Cassini Ion Neutral Mass Spectrometer: Analysis methods and comparison of measured ion densities to photochemical model simulations. J. Geophys. Res. 117, E10006 (2012)

K. Moe, B.R. Bowman, The effects of surface composition and treatment on drag coefficients of spherical satellites. Astrodynamics 123, 137 (2005)

I.C.F. Müller-Wodarg, R.V. Yelle, J. Cui, J.H. Waite, Horizontal structures and dynamics of Titan's thermosphere. J. Geophys. Res. 113, E10005 (2008)

M.E. Perry, B.D. Teolis, H.T. Smith, L.R. McNutt, G. Fletcher, W. Kasperzak, B. Magee, D.G. Mitchell, J.H. Waite, Cassini INMS observations of neutral molecules in Saturn's E-ring. J. Geophys. Res. 115, A10206 (2010)

M.S. Richard, T.E. Cravens, C. Wylie, D. Webb, Q. Chediak, R. Perryman, K.E. Mandt, J. Westlake, J.H. Waite, I.P. Robertson, B.A. Magee, N.J.T. Edberg, An empirical approach to modeling ion production rates in Titan's ionosphere. J. Geophys. Res. (2014). doi:10.1002/2013JA019706

F. Rizvi, Cassini thruster calibration algorithm using reaction wheel biasing data. J. Spacecr. Rockets 51, 563 (2014)

I.P. Robertson, T.E. Cravens, J.H. Waite, R.V. Yelle, V. Vuitton, A.J. Coates, J.E. Wahlund, K. Agren, K.E. Mandt, B.A. Magee, M.S. Richard, E. Fattig, Structure of Titan's ionosphere: Model comparisons with Cassini data. Planet. Space Sci. 57, 1834 (2009)

S. Sarani, A novel technique for the reconstruction of Titan atmospheric density using Cassini guidance, in AIAA Guidance, Navigation, and Control Conference, Hilton Head, South Carolina, USA, AIAA-20076343 (2007)

S. Sarani, Titan atmospheric density reconstruction using Cassini guidance, navigation, and control data, in AIAA Guidance, Navigation, and Control Conference, Chicago, AIAA 2009-5763 (2009)

D.A. Spencer, P.H. Kallemeyn, S.W. Thurman, Mars pathfinder entry, descent, and landing reconstruction. J. Spacecr. Rockets 36, 357 (1999)

J.R. Stalder, V.J. Zurick, Theoretical aerodynamic characteristics of bodies in a free molecular flow field. National Advisory Committee for Aeronautics. Technical Note 2423 (1951)

W. Steckelmacher, Knudsen flow 75 years on: the current state of the art for flow of rarefied gases in tubes and systems. Rep. Prog. Phys. 49, 1083 (1986)

D.F. Strobel, Molecular hydrogen in Titan's atmosphere: Implications of the measured tropospheric and thermospheric mole fractions. Icarus 208, 878 (2010)

D.F. Strobel, Hydrogen and methane in Titan's atmosphere: Chemistry, diffusion, escape, and the Hunten limiting flux principle. Can. J. Phys. 90, 795 (2012)

B.D. Teolis, M.E. Perry, B.A. Magee, J. Westlake, J.H. Waite, Detection and measurement of ice grains and gas distribution in the Enceladus plume by Cassini's Ion Neutral Mass Spectrometer. J. Geophys. Res. 115, A09222 (2010)

O.J. Tucker, R.E. Johnson, Thermally driven atmospheric escape: Monte Carlo simulations for Titan's atmosphere. Planet. Space Sci. 57, 1889 (2009)

O.J. Tucker, R.E. Johnson, J.I. Deighan, A.N. Volkov, Diffusion and thermal escape of H2 from Titan's atmosphere: Monte Carlo simulations. Icarus 222, 149 (2013)

E. Vigren, M. Galand, R. Yelle, J. Cui, J.E. Wahlund, K. Agren, P.P. Lavvas, I.C.F. Mueller-Wodarg, D.F. Strobel, V. Vuitton, A. Bazin, On the thermal electron balance in Titan's sunlit upper atmosphere. Icarus 223, 234 (2013)

V. Vuitton, R.V. Yelle, V.G. Anicich, The nitrogen chemistry of Titan's upper atmosphere revealed. Astrophys. J. 647, L175 (2006)

V. Vuitton, R. Yelle, M.J. McEwan, Ion chemistry and N-containing molecules in Titan's upper atmosphere. Icarus 191, 722 (2007) 
V. Vuitton, R.V. Yelle, J. Cui, Formation and distribution of benzene on Titan. J. Geophys. Res. 113, E05007 (2008)

J.H. Waite, W.S. Lewis, W.T. Kasperzak, V.G. Anicich, B.P. Block, T.E. Cravens, G.G. Fletcher, W.H. Ip, J.G. Luhmann, L.R. McNutt, H.B. Niemann, J.K. Parejko, J.E. Richards, P.C. Thomas, R.L. Thorpe, E.M. Walter, R.V. Yelle, The Cassini Ion and Neutral Mass Spectrometer (INMS) investigation. Space Sci. Rev. 114, 113 (2004)

A. Wellbrock, A.J. Coates, G.R. Lewis, J.H. Waite, Cassini CAPS-ELS observations of negative ions in Titan's ionosphere: Trends of density with altitude. Geophys. Res. Lett. 40, 4481 (2013)

E.P. Wenaas, Equilibrium cosine law and scattering symmetry at the gas-surface interface. J. Chem. Phys. 54, 376 (1971)

J.H. Westlake, J.M. Bell, R.E. Johnson, J.G. Luhmann, K.E. Mandt, B.A. Magee, A.M. Rymer, Titan's thermospheric response to various plasma environments. J. Geophys. Res. 116, A03318 (2011)

J.H. Westlake, J.H. Waite, J.M. Bell, R. Perryman, Observed decline in Titan's thermospheric methane due to solar cycle drivers. J. Geophys. Res.: Space Phys. 119, 8586 (2014)

R.V. Yelle, N. Borggren, V. de la Haye, W. Kasprzak, H.B. Niemann, I. Müller-Wodarg, J.H. Waite, The vertical structure of Titan's upper atmosphere from Cassini Ion Neutral Mass Spectrometer measurements. Icarus 182, 567 (2006)

R.V. Yelle, J. Cui, I.C.F. Müller-Wodarg, Methane escape from Titan's atmosphere. J. Geophys. Res. 113, E10003 (2008)

J.C. Zarnecki, B. Hathl, M. Leese, A. Ball, In-flight performance of the HASI servo accelerometer and implication for results at Titan, in International Workshop on Planetary Probe Atmospheric Entry and Descent Trajectory, Lisbon (2003), p. 71 